\title{
MANEJO QUIRÚRGICO ACTUAL DE LA ENFERMEDAD DE PEYRONIE GRAVE
}

\author{
Sava V. Perovic ${ }^{\dagger}$ y Rados P. Diinovic'.
}

Serbian Academy of Sciences and Arts. Belgrade. Serbia

'School of Medicine. University of Belgrade. Serbia.

Resumen.- OBJETIVO: Informar sobre los principios de la cirugía reconstructiva de diferentes deformidades del pene causadas por la enfermedad de Peyronie: restauración de la longitud, perímetro y forma con o sin implante de prótesis de pene.

MÉTODOS: En el período comprendido entre febrero 2007 y marzo de 2009, se realizó cirugía con parche por enfermedad de Peyronie en 98 pacientes con edades comprendidas entre 24 y 72 años (media 52 años). Las deformidades en el pene eran diferentes: curvatura dorsal en 54 (55\%) pacientes, lateral en 7 (7\%), ventral en $11(11 \%)$, y curvatura combinada en $21(21 \%) ; 24$ pacientes presentaban estrechamiento en el cuerpo cavernoso (reloj de arena) (24\%). Cuatro

\section{CORRESPONDENCIA}

Rados P. Djinovic

Sava Perovic Foundation

Borisavljeviceva, 58

11000 Belgrade (Serbia)

perovics@eunet.rs

Aceptado para publicar: 6 de noviembre 2009
(4\%) pacientes presentaban acortamiento de pene aislado, sin otras deformidades, y en dos (2\%) pacientes se encontró un estrechamiento cavernoso difuso aislado sin acortamiento. La gravedad de la curvatura varió de 60-90\%, media 72. Treinta y un (3 1\%) pacientes padecían disfunción eréctil asociada. Las opciones quirúrgicas para los casos graves de enfermedad de Peyronie fueron: injerto simple en 26 (26\%) pacientes, injertos complejos incluyendo incisión circular de la túnica albugínea en 36 (36\%), y los mismos procedimientos en pacientes con disfunción eréctil combinados con implante de prótesis de pene en 37 pacientes (38\%). La corrección quirúrgica se basó en la medición del defecto de la túnica y el cálculo preciso del tamaño del injerto y la forma. El enderezamiento y alargamiento del pene se logró, mediante la nivelación del lado/s acortados del pene con el más largo (convexo) y el injerto. El ancho del pene se restablece con incisión/es longitudinal/es adicionales e injertos; el ancho del injerto se determina mediante la medición de la diferencia en la circunferencia entre las partes normal y reducida del cuerpo cavernoso. En todos los casos, se utilizó InteXen LP (AMS) como material de injerto.

RESULTADOS: El seguimiento medio fue de 15 meses (6-25). El incremento de longitud del pene fue de 2,8 $\mathrm{cm}$ sin prótesis $(1,5-4,2 \mathrm{~cm})$ y $3,2 \mathrm{~cm}(2-4,5 \mathrm{~cm})$ con prótesis. En 5 pacientes (< 15 grados), la corrección de la curvatura fue insuficiente, debido a que el haz neurovascular fue un factor limitante. Veinticuatro pacientes informaron de hipoestesia y sensibilidad orgásmica reducida que se recuperó espontáneamente al cabo de 3-6 meses. Seis pacientes presentaron una disfunción eréctil de novo y 6 pacientes progresión de la enfermedad. Sólo un paciente con implante de prótesis de pene sufrió infección. El índice de satisfacción general de los pacientes fue del $95 \%$.

CONCLUSIONES: La reconstrucción de la túnica albugínea completa en la enfermedad de Peyronie puede 
realizarse como un procedimiento seguro por medio de injertos transversales, longitudinales y circulares con o sin implante simultáneo de prótesis de pene. Se puede lograr, mediante el cálculo geométrico, la longitud máxima del pene, el grosor y la recuperación de la forma, independientemente del tipo de deformidad.

Palabras clave: Pene. Enfermedad de Peyronie. Cirugía. Principio geométrico. Injerto. InteXen LP.

Summary.- OBJECTIVES: To report the principles of penile re-sculpturing of different deformities caused by M. Peyronie: restoration of penile length, girth and shape with or without penile prosthesis implantation.

METHODS: In the period between February 2007 and March 2009, we performed grafting surgery for M. Peyronie in 98 patients aged between 24 and 72 years (mean 52 years). Penile deformities were diferent: dorsal curvature in $54(55 \%)$, lateral in $7(7 \%)$, ventral in $11(17 \%)$, and combined curvature in $21(21 \%)$; associated corporal narrowing was present in 24 patients (24\%). Four (4\%) patients presented isolated penile shortening without other deformity. Isolated diffuse corporal narrowing without shortening was found in two (2\%) patients. Severity of curvature ranges from 60 to 90 degrees, mean 72 . Thirty one (37\%) patients had associated ED. Surgical options for severe Peyronie's disease were: single grafting in 26 pts (26\%), complex grafting including circular tunical incision in 36 pts (36\%), and in patients with ED the same procedures combined with penile prosthesis implantation (37 pts, 38\%). Surgical correction was based on measurement of the tunical defect and precise calculation of graft size and shape. Penile straightening and lengthening was achieved by equalizing of shortened penile side/s with the longest one (convex) and grafting. Penile width is reestablished with additional longitudinal incision/s and grafting; graft width is determined by measurement of difference in circumference between normal and narrowed part of the corpora. We used Intexen LP (AMS) as a grafting material in all cases.

RESULTS: The mean follow-up was 15 months (6-25). Mean penile length gain without prosthesis was $2.8 \mathrm{~cm}$ (1.5-4.2) and with prosthesis $3.2 \mathrm{~cm}(2-4.5 \mathrm{~cm})$. Insuficient straightening was in 5 patients />15 degree) where Neuro Vascular Bundle (NVB) was limiting factor. Twenty four patients reported hypoesthesia and reduced orgasmic sensitivity that recovered spontaneously after 3-6 months. De-novo ED occurred in 6 pts and progression of disease in 6 patients. Infection occurred only in one patient with penile prosthesis implantation. Overall patients' satisfaction was $95 \%$.
CONCLUSIONS: Complete tunical reconstruction in IPP can be performed as a safe procedure by transversal, longitudinal and circular grafting with or without simultaneous penile prosthesis implantation. Maximum penile length, girth and shape restoration can be achieved using geometrical calculation, regardless of type of deformity.

Keywords: Penis. Peyronie's disease. Surgery. Geometrical principle. Grafting. Intexen LP.

\section{INTRODUCCIÓN}

La enfermedad de Peyronie se caracteriza por diferentes deformidades en el pene causadas por la fibrosis y contracción de la túnica albugínea. La enfermedad se asocia casi siempre con el acortamiento del pene. Tiene gran influencia en la calidad de vida y a menudo se relaciona con importantes consecuencias psicológicas. La incidencia es del 3$10 \%$ en la población masculina, y es frecuente en el rango entre 40 y 70 años, sin embargo también puede afectar a población joven menor de 30 años $(1,2)$. La enfermedad de Peyronie se asocia comúnmente con disfunción eréctil (DE), que oscila desde el 20 hasta el $54 \%$ según Usta et al. (3).

La indicación de tratamiento quirúrgico requiere que la enfermedad se encuentre en fase estable. El concepto de enfermedad estable no se ha definido claramente, pero generalmente se acepta el padecer la enfermedad durante al menos un año, que hayan transcurrido por lo menos seis meses sin ningún cambio en la deformidad y que se haya resuelto el dolor. La progresión de la enfermedad es incierta, y no hay ningún parámetro fidedigno que lo prediga. El candidato a cirugía también debe presentar, generalmente, la imposibilidad de realizar el coito como consecuencia de la deformidad o rigidez inadecuada. Además, es un candidato adecuado para cirugía un paciente al que le ha fallado el tratamiento médico y tenga una calcificación extensa de la placa. Por último, el paciente que desee resultados más rápidos y fiables debe escoger un tratamiento quirúrgico.

El tratamiento quirúrgico debe ser individualizado, con el objetivo no sólo de restaurar la función del pene, sino también la recuperación en la medida de lo posible, del estado previo del paciente. En el pasado, se han propuesto diferentes técnicas y se sugirieron diferentes materiales para el injerto y para cubrir el defecto de la túnica, pero en la actualidad, ninguna de ellas representa el "gold standard". 
Nuestro objetivo es presentar una experiencia personal en el tratamiento quirúrgico de la enfermedad de Peyronie grave basado en el principio geométrico, es decir, la restauración completa de la longitud del pene, el grosor y forma, con o sin implante de una prótesis peneana.

\section{MATERIALES Y MÉTODOS}

Entre febrero de 2007 y marzo del 2009 hemos realizado cirugía, basada en la medición geométrica, en 98 pacientes con enfermedad de Peyronie grave, utilizando injerto de matriz de colágeno acelular de porcino (InteXen LP, AMS) para la reconstrucción de la túnica albugínea. El promedio de edad es de 52 años (24 a 72). Las deformidades en el pene fueron: dorsal en $54(55 \%)$, lateral en $7(7 \%)$, ventral en $11(11 \%)$, y curvatura combinada en $21(21 \%)$; estrechamiento del cuerpo cavernoso en 24 (27\%). Curvatura en más de una localización se presentó en $15(16 \%)$ pacientes. Cuatro (4\%) pacientes tenían sólo acortamiento del pene sin otras deformidades. Dos pacientes $(2 \%)$ presentan estrechamiento difuso del cuerpo cavernoso. La gravedad de la curvatura varió desde 60 hasta 90 grados, media 72 . Treinta y un (31\%) pacientes padecían DE asociada (Tabla I).

TABLA I. TIPOS DE DEFORMIDADES GRAVES DE PENE Y DE ASOCIADA.

\begin{tabular}{|l|c|}
\hline & $\begin{array}{c}\text { Deformidades } \\
\text { graves }\end{array}$ \\
\hline Curvatura Dorsal & 54 pts. (55\%) \\
\hline Curvatura Lateral & 7 pts. (7\%) \\
\hline Curvatura Ventral & 11 pts. (11\%) \\
\hline Curvatura Combinada & 21 pts. (21\%) \\
\hline Estrechamiento Asociado & 24 pts. (25\%) \\
\hline Mas de una Curvatura & 15 pts. (15\%) \\
\hline Acortamiento Aislado del pene & 4 pts. (4\%) \\
\hline Estrechamiento Aislado del pene & 2 pts. (2\%) \\
\hline DE Asociada & 37 pts. (38\%) \\
\hline
\end{tabular}

La indicación para la cirugía fue la deformidad grave del pene que hacia la relación sexual difícil o imposible con síntomas durante más de 12 meses, y enfermedad estable durante más de seis meses.

La valoración preoperatoria incluyó la historia clínica completa, así como la evaluación de las comorbilidades. La evaluación de la deformidad del pene, la erección y la rigidez se realizó mediante cuestionario IIEF-5 (5) y objetivamente mediante inyección intracavernosa (ICI) de alprostadil (10-20ug). Todos los pacientes que tenía DE moderada a grave (37 pts.), basado en la puntuación de los resultados del IIEF-5 (5-1 1) y la prueba del ICI fueron candidatos a cirugía con implante de prótesis de pene. Los pacientes, cuya resultados del cuestionario IIEF5 fue entre 24 y 25 con prueba normal de $\mathrm{ICl}$, se consideraron para cirugía sin implante de prótesis de pene (54 pacientes). Se utilizó también ecografía doppler color para la evaluación vascular (insuficiencia arterial y/o disfunción veno-oclusiva), así como para la detección y localización de vasos colaterales entre las arterias dorsal y cavernosa.

\section{Alternativas quirúrgicas}

Las técnicas quirúrgicas se seleccionan de acuerdo a la determinada forma geométrica del defecto de la túnica albugínea después de la incisión/ es. El objetivo de la cirugía fue la restauración de la forma del pene con un promedio de enderezamiento, prolongación y ampliación con el fin de satisfacer las expectativas de los pacientes tanto como sea posible.

Después de denudar el pene, el haz neurovascular es ampliamente movilizado comenzando parauretralmente con el fin de alcanzar una longitud suficiente después del enderezado y alargamiento de la túnica albugínea. También se movilizó la uretra en los casos con curvatura ventral o lateral. Cuando la placa implica al haz neurovascular, podría ser difícil encontrar el plano correcto de disección y se debe tener especial cuidado en no lesionar sus elementos. Su elasticidad se reduce y puede ser un factor limitante para el adecuado alargamiento del pene. Se induce una erección completa artificial con PGE 1 o inyección intracavernosa de solución salina después de la colocación de torniquete para estimar la deformidad. Se aplicaron varias técnicas quirúrgicas en función de la deformidad encontrada: injerto sencillo en 59 pts. $(60 \%)$, injertos complejos incluyendo incisión tunicular circular en 39 pts. (40\%); la implantación de prótesis de pene simultánea se realizó en 37 pts. $(38 \%)$ con DE. 


\section{Procedimiento de injerto sencillo}

La indicación para este tipo de cirugía es una curvatura del pene situada en un punto. El ancho del defecto tunical, es decir, el injerto es igual a la diferencia entre el lado convexo más largo y el cóncavo más corto; su longitud se determina midiendo la distancia entre dos líneas circulares marcadas perpendicularmente a las partes proximal y distal normal, no afectadas de la túnica. El nivel de la incisión tunical transversal es en los puntos de intersección de los ejes proximal y distal marcados en los lados laterales del cuerpo cavernoso (Figura 1A). La incisión debe pasar la mitad de la circunferencia con incisiones de relajación en " $Y$ " en los bordes para permitir el contorno recto después del injerto. De esta forma, la incisión lineal se transforma en defecto cuadrangular o hexangular. La albugínea se ve socavado por la movilización del tejido cavernoso con tabique de $2-3 \mathrm{~cm}$ proximal y distal con el fin de permitir su estiramiento y una mejor redistribución en el marco del injerto (Figura 1B-D). En el caso de placa calcificada, se extirpa cuidadosamente, conservando la fina capa de la túnica no afectada.

La forma de defecto tunical, es decir, el injerto fue cuadrangular o hexagonal, en función del grado de curvatura y la longitud de las extensiones en " $Y$ ". Cuando es hexagonal, la anchura máxima del injerto se corresponde con el punto de máxima curvatura. La longitud del injerto fue calculado por sustracción de una parte sin extirpar túnica de la circunferencia. En la curvatura ventral, la incisión circular recorre los lados ventral y lateral con extensiones dorsales en "Y" (Figura 2A-D). En la curvatura aislada lateral, la incisión se inicia en la uretra, pasa sobre el lado afectado hasta surco dorsal (Figura 3A-D).

En todos los casos se utilizó matriz dérmica porcina acelular de colágeno (InteXen LP, AMS) como material de injerto. Antes de su uso, se deshidrata y se sumerge en solución salina durante 5 minutos. La forma del injerto se creó de acuerdo con el cálculo descrito. Se sutura a la túnica utilizando sutura continua PDS 4-0, dando la vuelta hacia dentro a la superficie lisa del injerto. También se cose a la membrana mediante varias suturas de colchonero evitando protuberancias y permitiendo un mejor contacto con el tejido cavernoso en el primer período postoperatorio. El enderezamiento y alargamiento del pene se estimó con erección artificial con suero salino (Figura 1D, 2D, 3D).

\section{Injerto Complejo}

Este tipo de injerto está indicado en los casos con deformidad compleja del pene: curvaturas en más de un punto, curvaturas en más de un plano (es decir, dorso lateral, ventrolateral), curvatura del pene combinada con estrechamiento, localización apical de la placa (glande flácido) y acortamiento o retracción de cuerpo cavernoso aislada.

En los casos en que la curvatura se encuentra en el mismo plano con dos puntos de curvatura len el mismo o en los lados opuestos del pene - pene en forma de S) se injerta en ambos lugares usando el principio geométrico descrito (Figura 4A, B). El ancho proximal del injerto se calcula como la diferencia entre el lado convexo y cóncavo de la parte proximal y media normal del pene, y la anchura distal del injerto como la diferencia entre el lado convexo y cóncavo de la parte media y distal normal del pene.

Cuando la curvatura es combinada, es decir, en más de un punto de un plano, se utilizó otro principio. Se marcan las partes normales proximal y distal con dos líneas circulares perpendiculares y se mide la distancia entre ellas en varios puntos alrededor de la circunferencia, todos ellos paralelos a las fibras longitudinales albugíneas. El más largo, determina la longitud convexa y el objetivo es alargar todas las partes acortadas de la albugínea a la misma medida. El punto de contracción máxima estaba en el lado dorso o ventro-lateral, donde el injerto fue también más amplio. La forma del injerto fue hexagonal irregular.

Cuando la curvatura del pene se combinó con estrechamiento del mismo, se combinó el injerto transversal (descrito) con una incisión longitudinal proximal y distal con relajación en los extremos del injerto. De esta manera se consiguió la ampliación simultánea del pene. El ancho longitudinal del injerto se determina como la diferencia entre la circunferencia normal y la circunferencia del cuerpo cavernoso retraído. La longitud del injerto se corresponde con la longitud de la zona estenótica. Los injertos se suturan entre sí con sutura continua Prolene 40 , para evitar la dehiscencia postoperatoria (Figura 5A-C).

Si la placa se encuentra debajo del glande en la parte superior de los cuerpos cavernosos, se realizó un desmontaje total del pene, que permite el abordaje del extremo distal de los cuerpos cavernosos deformados. El reestablecimiento de la correcta anatomía del extremo distal de los cuerpos cavernosos se realiza mediante una incisión longitudinal y/o transversal, separando el tejido cavernoso y el injerto utilizando el mismo principio geométrico que el de la restauración de la longitud/circunferencia del pene. (Figura 6A-E). 


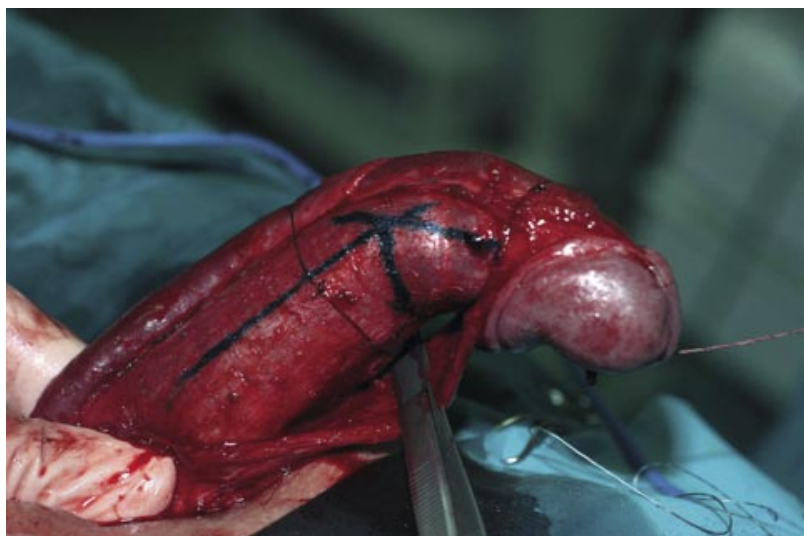

FIGURA 1. Injerto Sencillo - Curvatura dorsal: A). Medida geométrica del defecto de la túnica albugínea. Punto de máxima curvatura en la intersección de dos líneas paralelas al eje del pene. Determinación del ancho del injerto, midiendo la diferencia en longitud del lado más largo y el más corto fuera del cuerpo afectado. La longitud del injerto equivalente a la longitud de la incisión circular, con un final bifurcado cerca de la uretra.

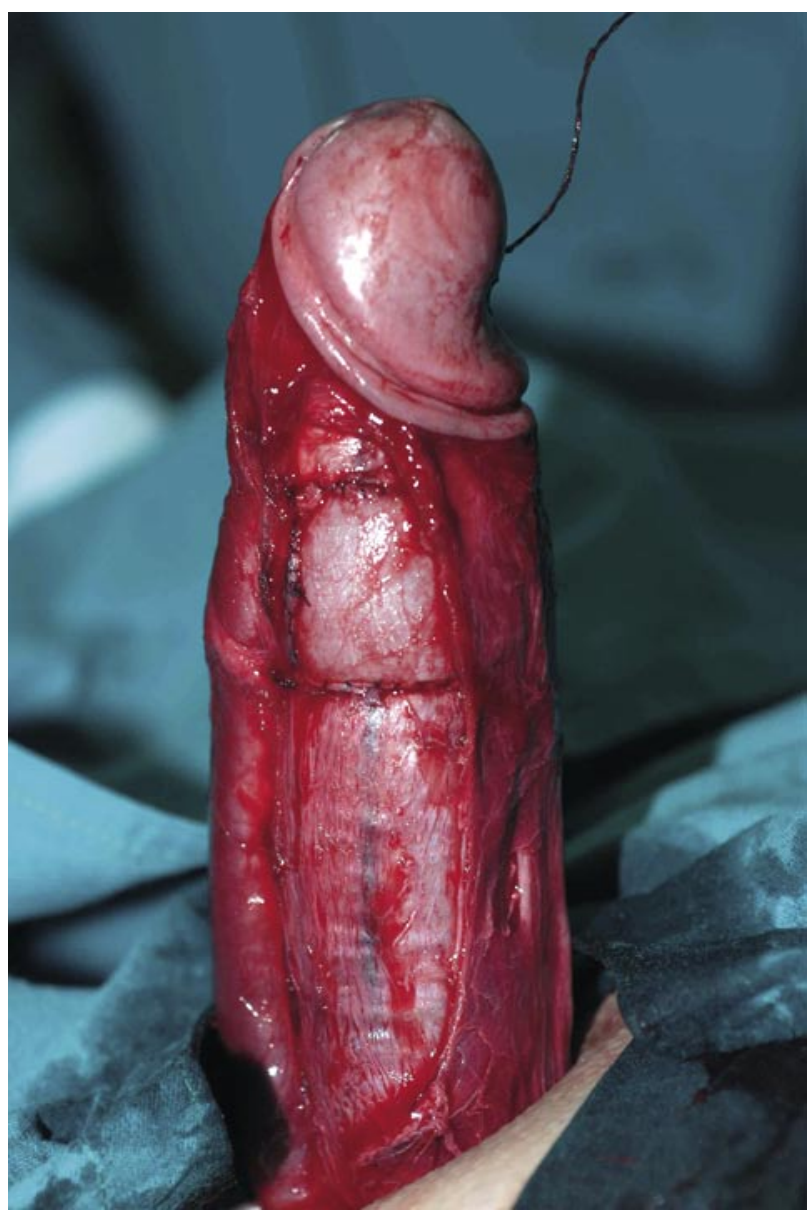

FIGURA 1C). Injerto con Intexen LP.

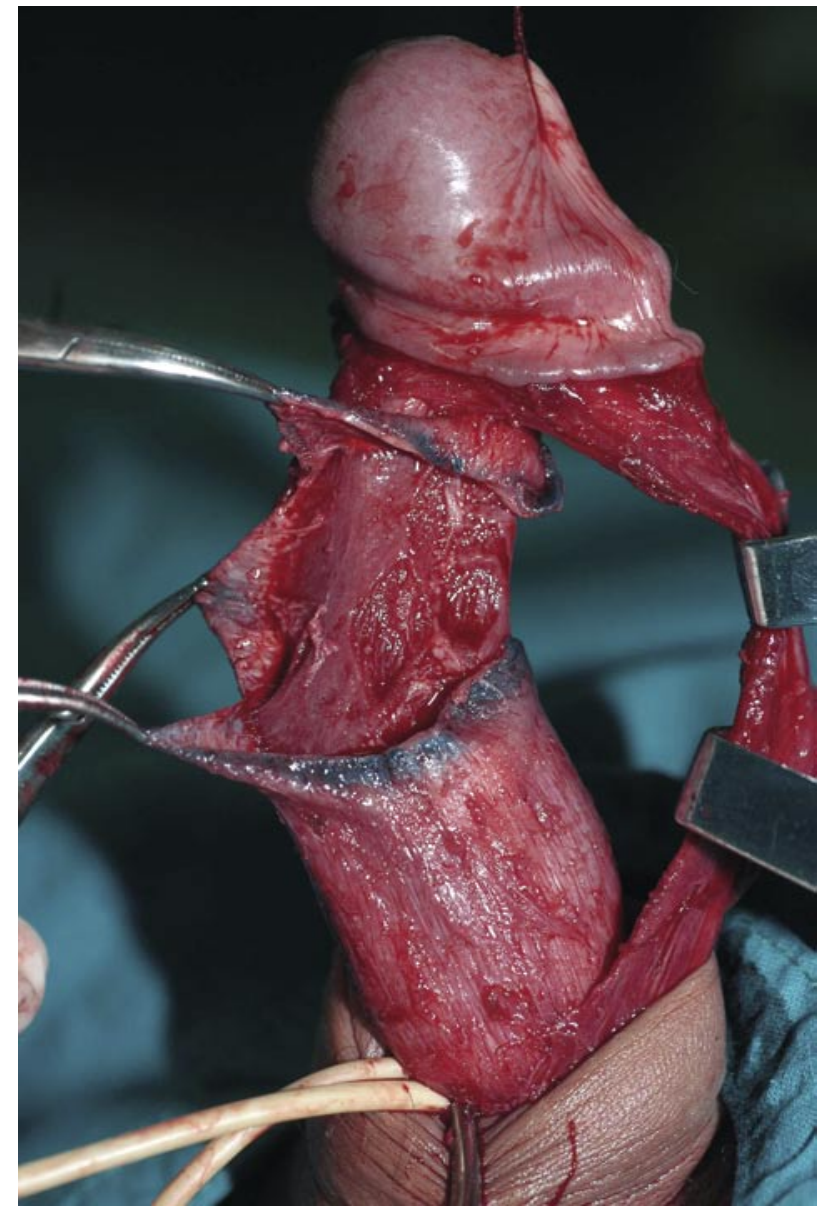

FIGURA 1B). El defecto de la túnica está creado y se ha separado el tejido eréctil.

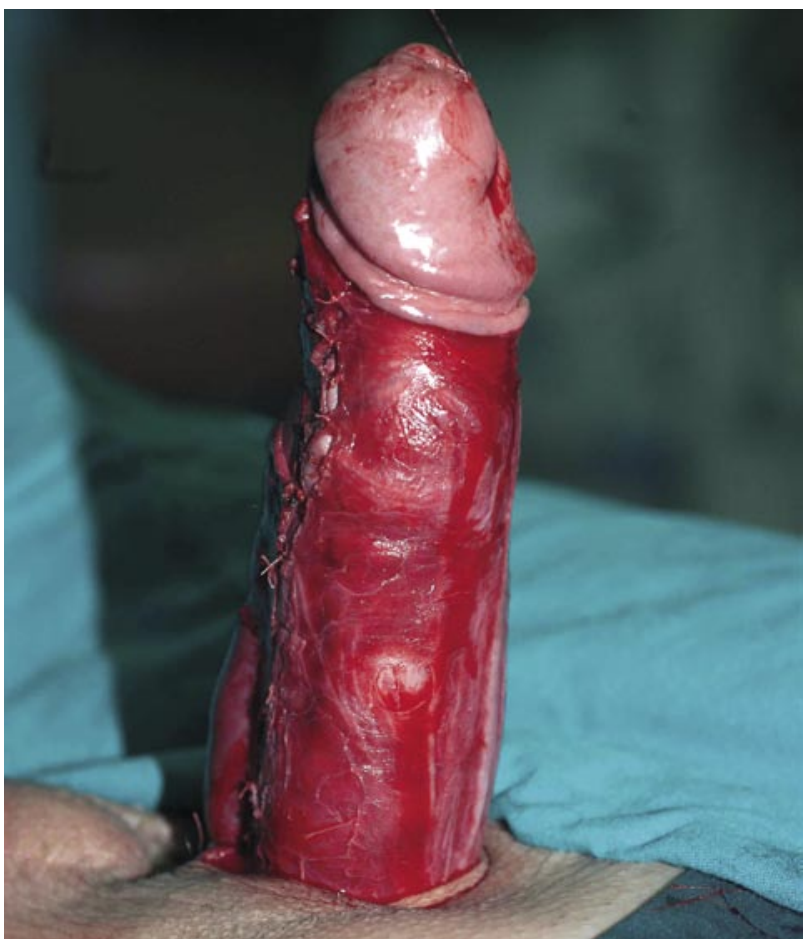

FIGURA 1D). Alargamiento y enderezamiento completo en erección total. 


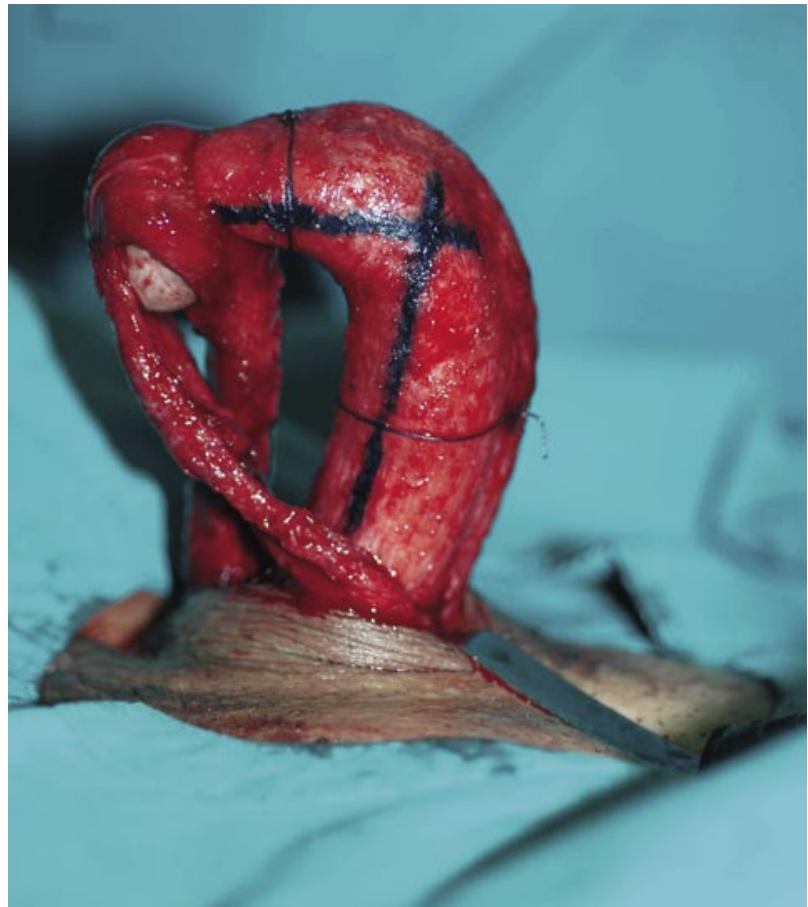

FIGURA 2. Injerto Sencillo - Curvatura ventral: A). Curvatura ventral grave.

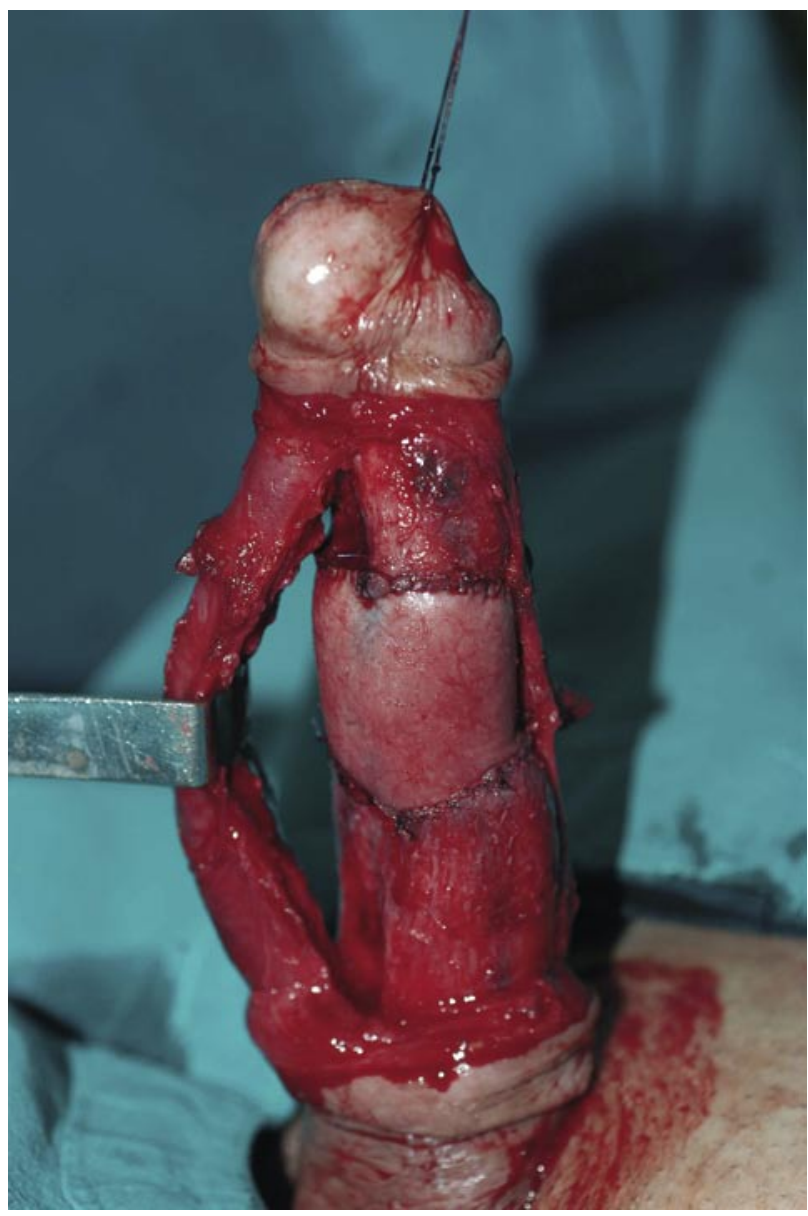

FIGURA 2C). La erección muestra el alargamiento y enderezamiento del pene.

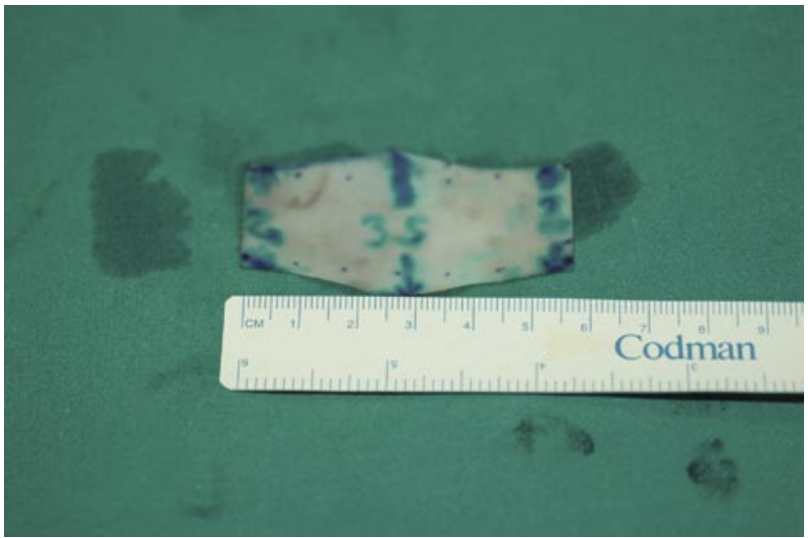

FIGURA 2B). Injerto en forma exagonal.

\section{Implante de Prótesis de Pene}

Los pacientes con enfermedad de Peyronie y DE que no respondieron a tratamiento conservador fueron candidatos para la implante de prótesis de pene. Primero, los pacientes se sometieron a re-

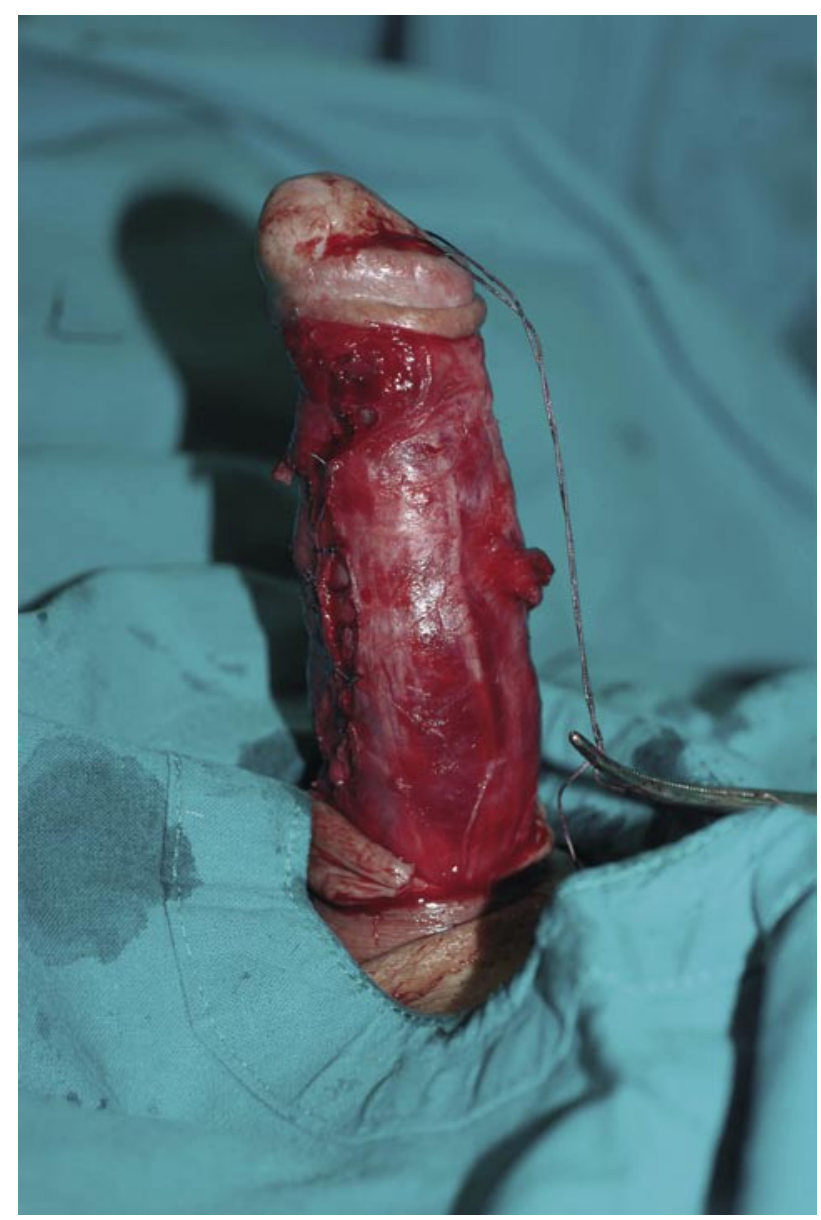

FIGURA 2D). 


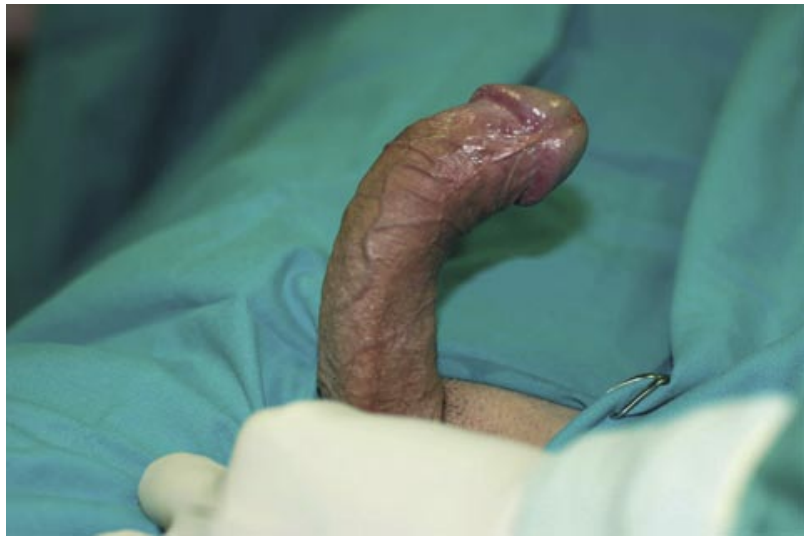

FIGURA 3. Injerto Sencillo - Curvatura lateral: A) Curvatura lateral.

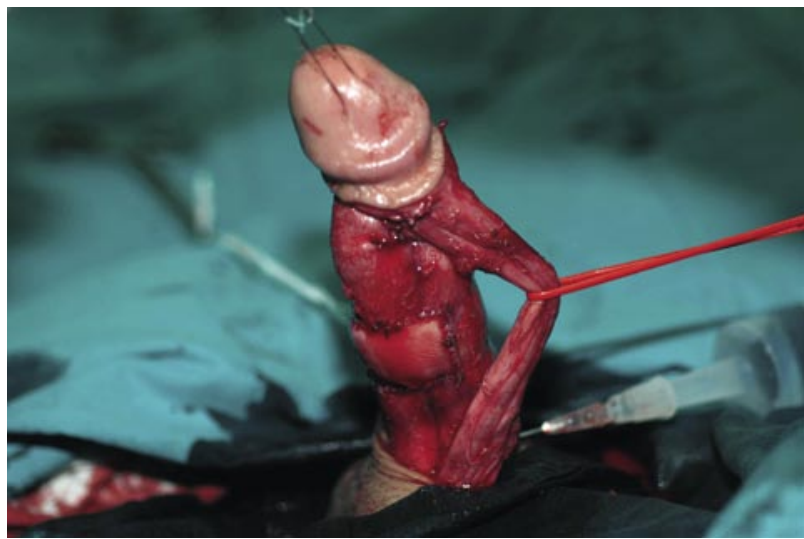

FIGURA 3C). Injerto en una corpora cavernosa afectada por una enfermedad.

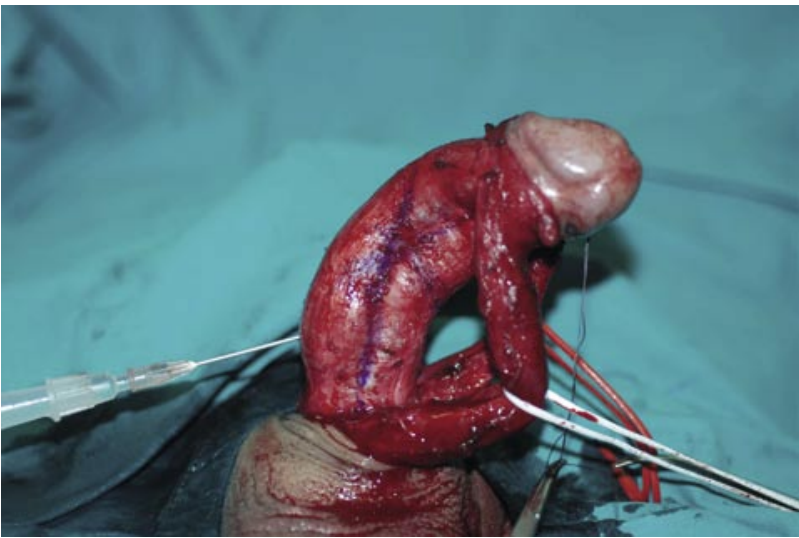

FIGURA 3B). Movilización de la uretra y haz neurovascular.

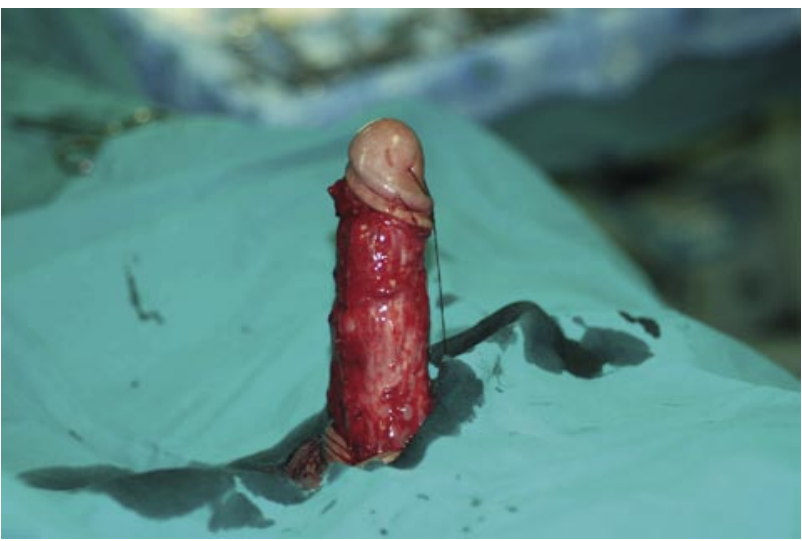

FIGURA 3D). Estiramiento completo peneano en maxima erección.

TABLA II. RESULTADOS DE LA RECONTRUCCIÓN MEDIANTE PROCEDIMIENTOS DE INIERTO,

UTILIZANDO EL PRINCIPIO GEOMÉTRICO, CON O SIN PRÓTESIS.

\begin{tabular}{|c|c|c|c|c|c|c|c|}
\hline $\begin{array}{c}\text { Tipo de } \\
\text { procedimiento }\end{array}$ & $\begin{array}{l}\text { №. } \\
\text { De pts }\end{array}$ & Seguimiento & $\begin{array}{l}\text { Incremento } \\
\text { longitud }\end{array}$ & $\begin{array}{l}\text { Re-curvatura } \\
<15 \text { grados }\end{array}$ & $\begin{array}{c}\text { De novo } \\
\text { DE }\end{array}$ & $\begin{array}{l}\text { Progresión de } \\
\text { la enfermedad }\end{array}$ & Infección \\
\hline $\begin{array}{l}\text { Injerto } \\
\text { simple }\end{array}$ & $\begin{array}{l}59 \text { pts. } \\
(60 \%)\end{array}$ & \multirow{3}{*}{$\begin{array}{c}\text { 6-25 meses } \\
\text { (media } 15 \\
\text { meses) }\end{array}$} & \multirow{2}{*}{$\begin{array}{c}2.8 \mathrm{~cm} \text { (rango } \\
1.5-4.2 \mathrm{~cm} \text { ) }\end{array}$} & 2 pts $(2 \%)$ & 2 pts $(2 \%)$ & 2 pt $(2 \%)$ & / \\
\hline $\begin{array}{l}\text { Injerto } \\
\text { complejo }\end{array}$ & $\begin{array}{l}39 \text { pts } \\
(40 \%)\end{array}$ & & & 3 pts $(3 \%)$ & 4 pts (4\%) & 4 pts $(4 \%)$ & / \\
\hline $\begin{array}{l}\text { Injerto con pró- } \\
\text { tesis de pene }\end{array}$ & $\begin{array}{l}37 \text { pts } \\
(38 \%)\end{array}$ & & $\begin{array}{c}3.2 \mathrm{~cm} \text { (rango } \\
2-4.5 \mathrm{~cm} \text { ) }\end{array}$ & / & / & / & $1 \mathrm{pt}(1 \%)$ \\
\hline Total & 98 & / & / & 5 pts $(5 \%)$ & 6 pts $(6 \%)$ & $6 \operatorname{pts}(6 \%)$ & $1 \mathrm{pt}(1 \%)$ \\
\hline
\end{tabular}


TABLA III. PACIENTES Y SATISFACCIÓN PAREJA

\begin{tabular}{|c|c|c|c|c|c|}
\hline \multirow{2}{*}{$\begin{array}{l}\text { Grado de } \\
\text { satisfacción }\end{array}$} & \multicolumn{2}{|c|}{$\begin{array}{c}\text { Satisfacción de los Pacientes con la } \\
\text { vida sexual }\end{array}$} & \multicolumn{2}{|c|}{$\begin{array}{c}\text { Satisfacción de las Parejas con la } \\
\text { vida sexual }\end{array}$} & \multirow{2}{*}{$\begin{array}{l}\text { Satisfacción general } \\
\text { de los Pacientes con } \\
\text { el resultado de la } \\
\text { cirugía }\end{array}$} \\
\hline & $\begin{array}{l}\text { Sin } \\
\text { prótesis }\end{array}$ & $\begin{array}{l}\text { Con } \\
\text { prótesis }\end{array}$ & $\begin{array}{l}\text { Sin } \\
\text { prótesis }\end{array}$ & $\begin{array}{l}\text { Con } \\
\text { prótesis }\end{array}$ & \\
\hline Muy Satisfecho & 36 pts $(59 \%)$ & 27 pts $(74 \%)$ & 32 pts $(53 \%)$ & 34 pts (91\%) & 63 pts (64\%) \\
\hline Satisfecho & 20 pts $(33 \%)$ & 9 pts (24\%) & 24 pts $(40 \%)$ & 3 pts $(9 \%)$ & 30 pts (31\%) \\
\hline Descontentos & 5 pts $(8 \%)$ & 1 pts (2\%) & 5 pts $(7 \%)$ & / & 5 pts $(5 \%)$ \\
\hline
\end{tabular}

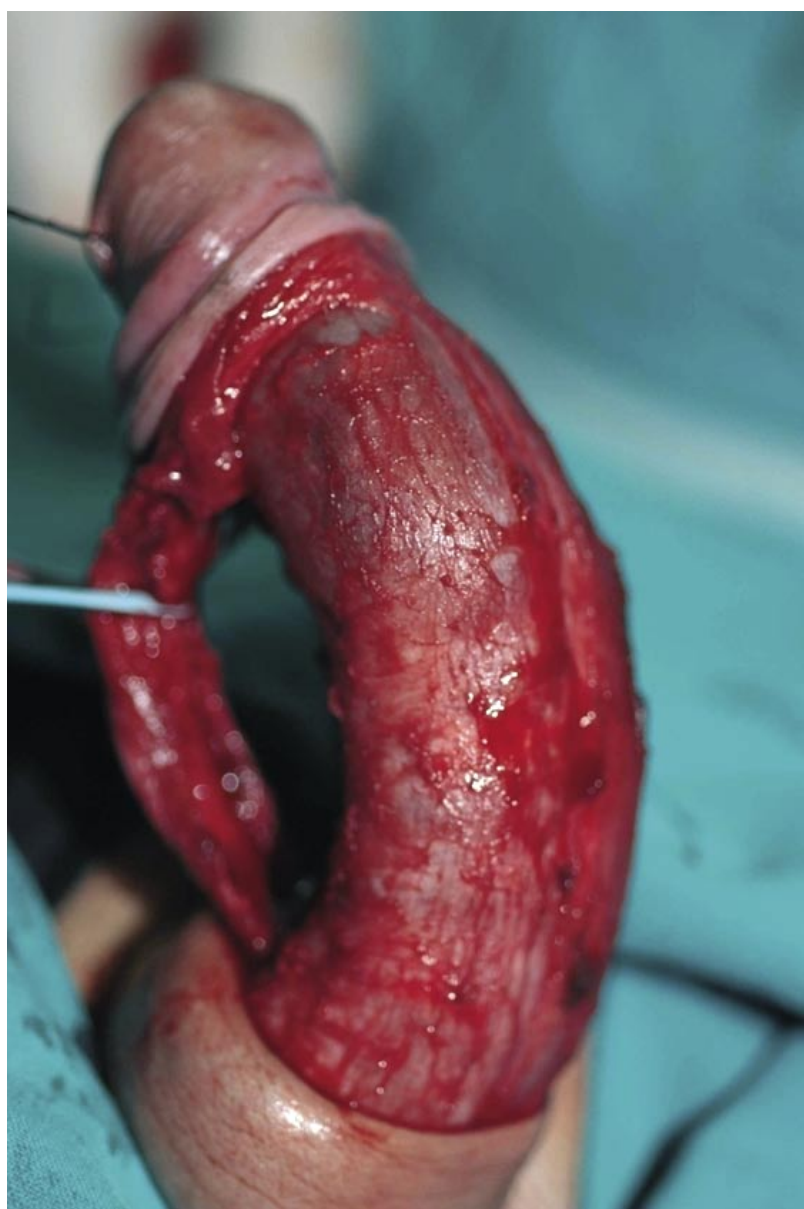

FIGURA 4. Injerto Complejo - Curvatura dorsal doble: A). Las dos fleches muestran los puntos de las dos curvaturas.

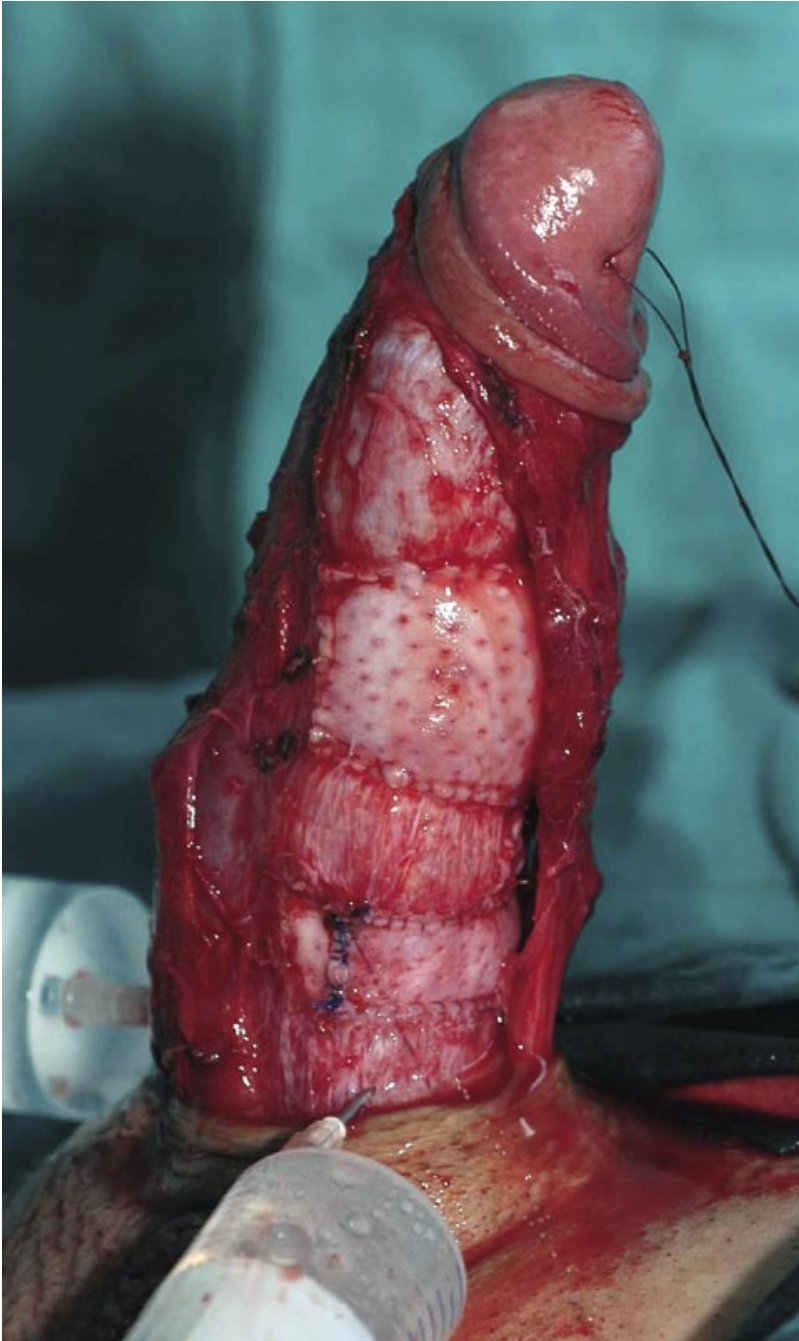

FIGURA 4B). Injerto doble del defecto tunical. La erección artificial mostró curvatura. 


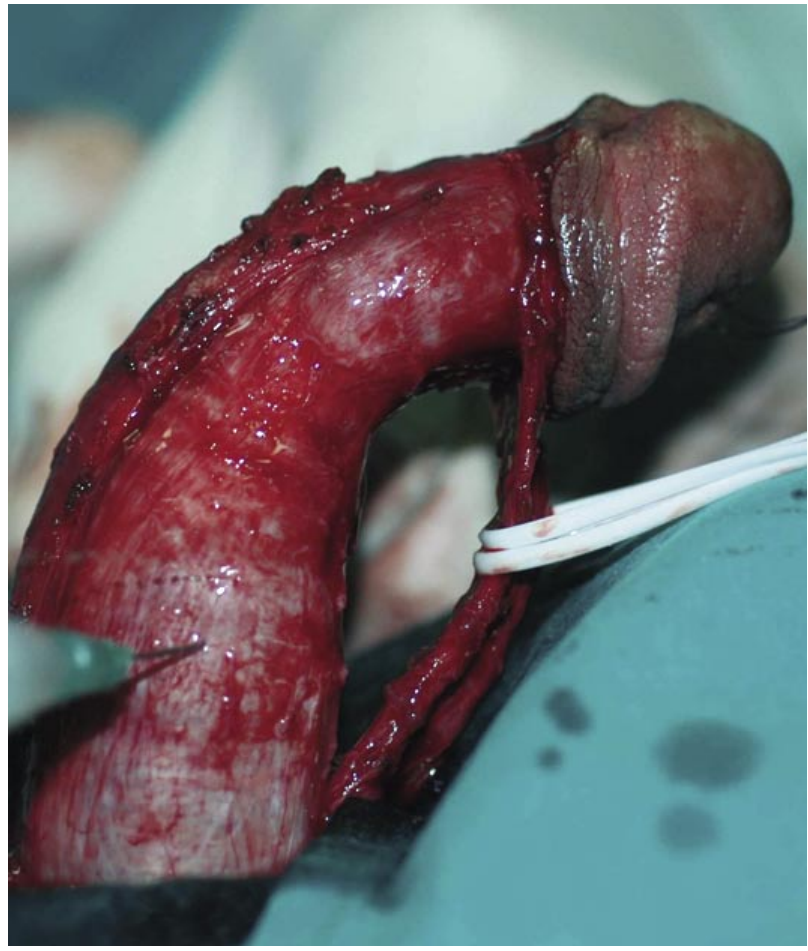

FIGURA 5. Injerto complejo de curvatura dorsal y distal y estrechamiento proximal:

A). Curvatura dorsal, con largo fenómeno de estrechamiento.

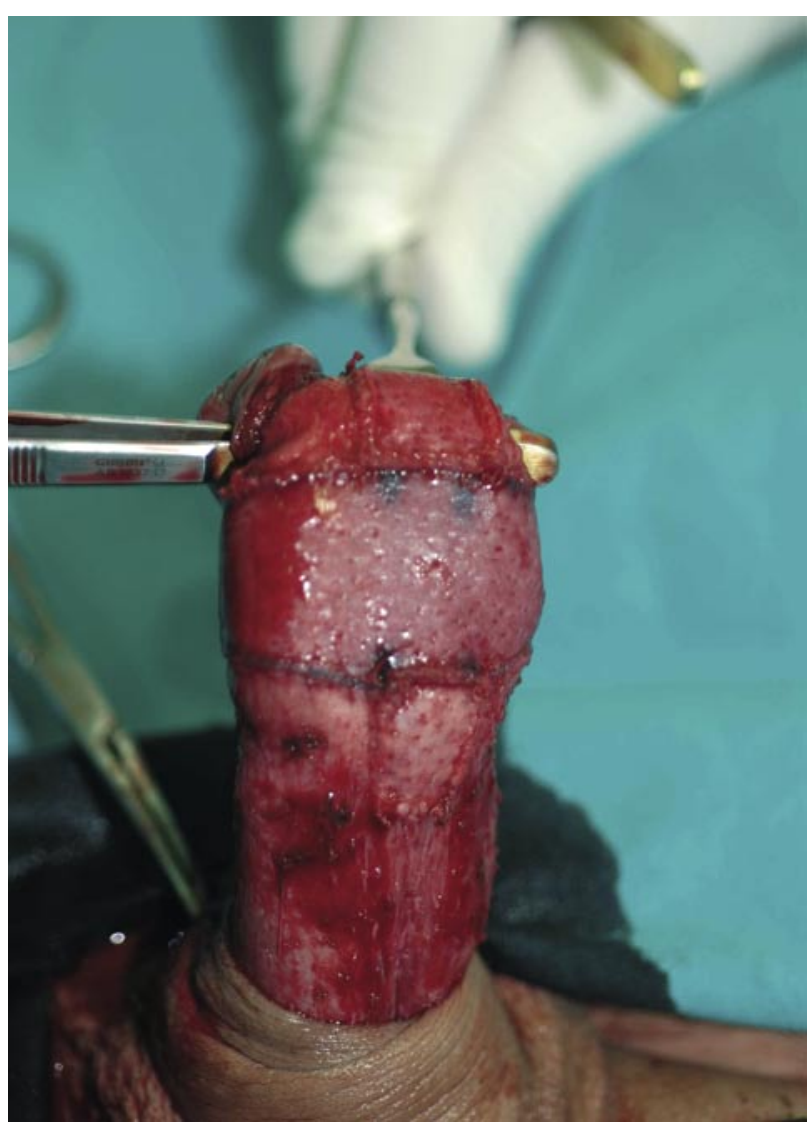

FIGURA 5B). Injerto combinado con uno transversal y dos longitudinales. construcción de la longitud, grosor y forma del pene utilizando los criterios descritos y, a continuación implante de prótesis de pene con procedimiento estándar (Figura 7A-D). Se realizó incisión circular a los pacientes con enfermedad difusa y acortamiento importante de la túnica albugínea del pene, separando e injertando el tejido cavernoso (Figura 8A, B).

En nuestra opinión, es mejor realizar dos incisiones circulares completas en dos diferentes niveles que una, para evitar estiramiento del tejido cavernoso, que se produce si sólo se realiza una incisión circular (Figura 9A-F). Se implantan dos o tres piezas de prótesis de pene inflable o maleable, con el tamaño compatible con el pene alargado. El alargamiento del pene se limita siempre por la longitud y elasticidad del haz neurovascular, ya que la uretra y el tejido cavernoso se estiran fácilmente.

Se utilizan dos injertos laterales longitudinales para estrechar los cuerpos cavernosos aislados y mejorar el contorno (Figura 10A-C).

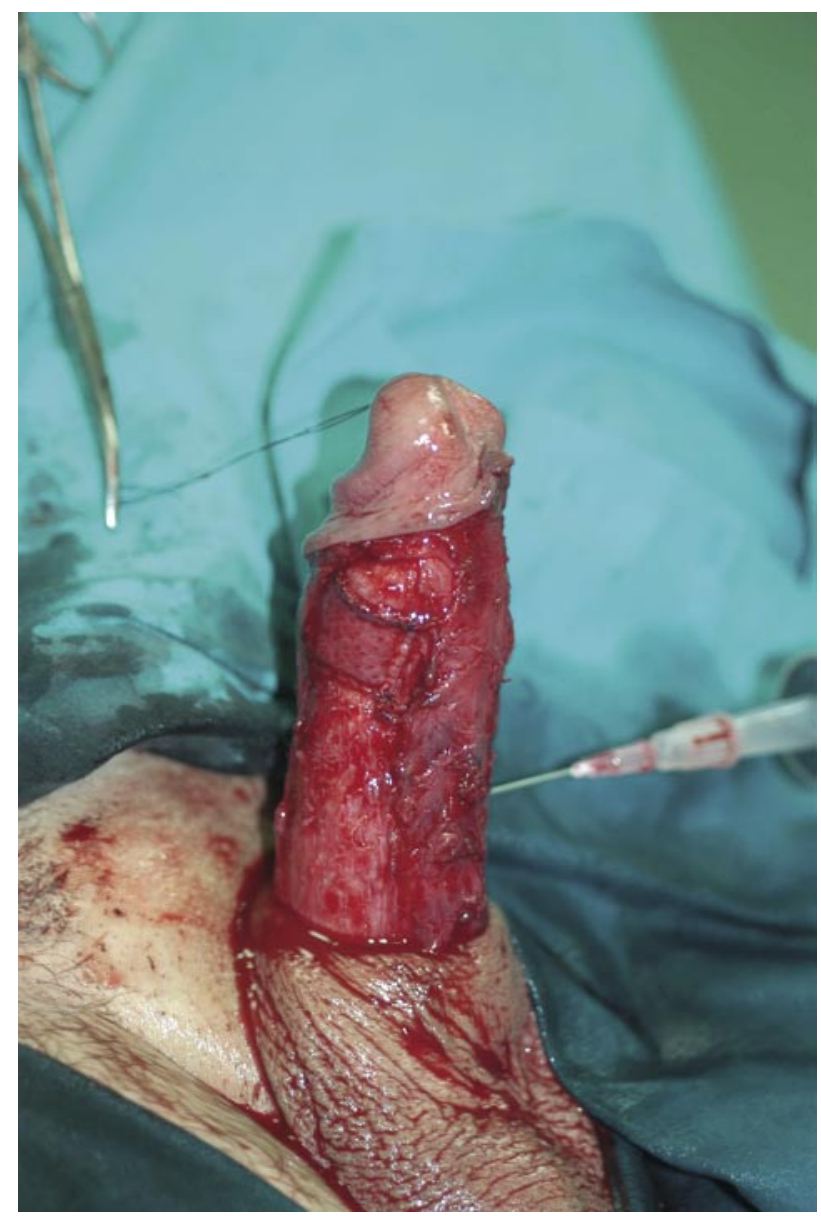

FIGURA 5C). Enderezamiento y ensanchamiento completo del pene. 


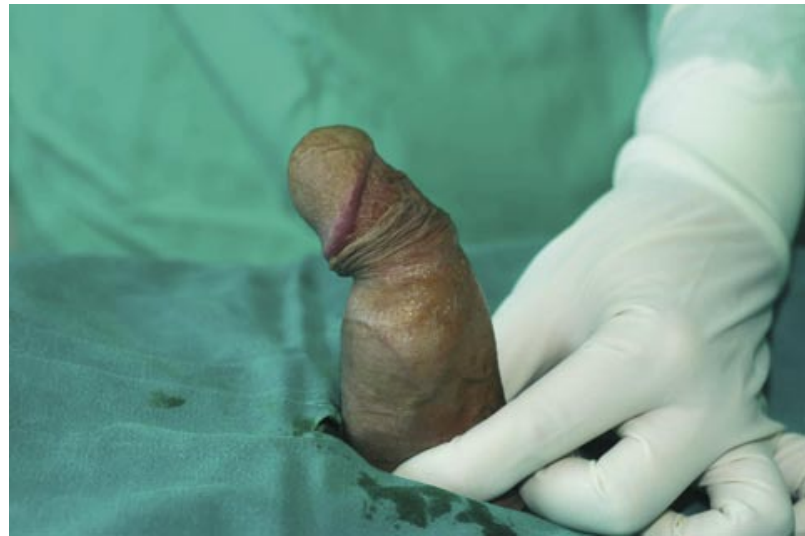

FIGURA 6. Injerto Complejo - Deformidad "Floppy glans":

A). Curvatura dorsal con excesivo estrechamiento distal del cuerpo.

\section{Fisioterapia postoperatoria}

Con el fin de inducir la erección y aumentar el flujo sanguíneo del pene, se prescribieron inhibidores PDE5 diarios durante 3-4 semanas a partir de 2-3 días después de la cirugía.

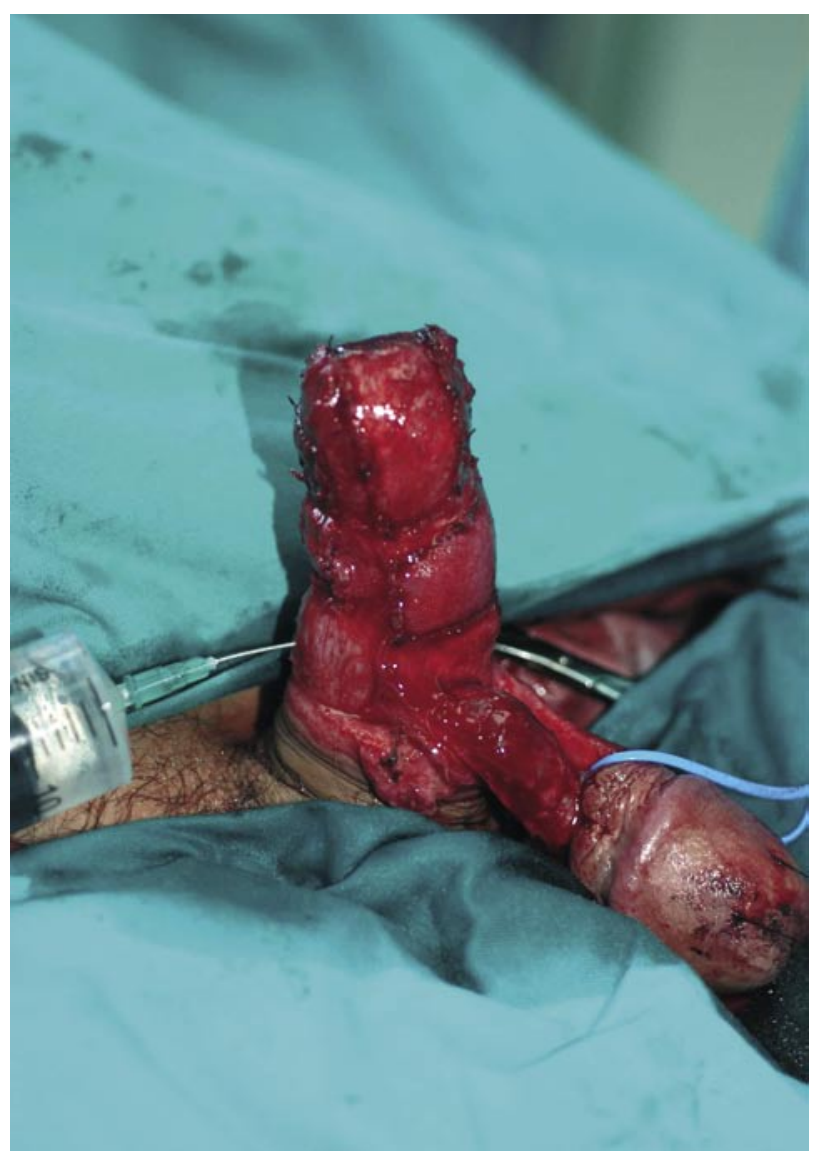

FIGURA 6C.) Restauración de la parte distal del cuerpo combinando injerto longitudinal y transversal.

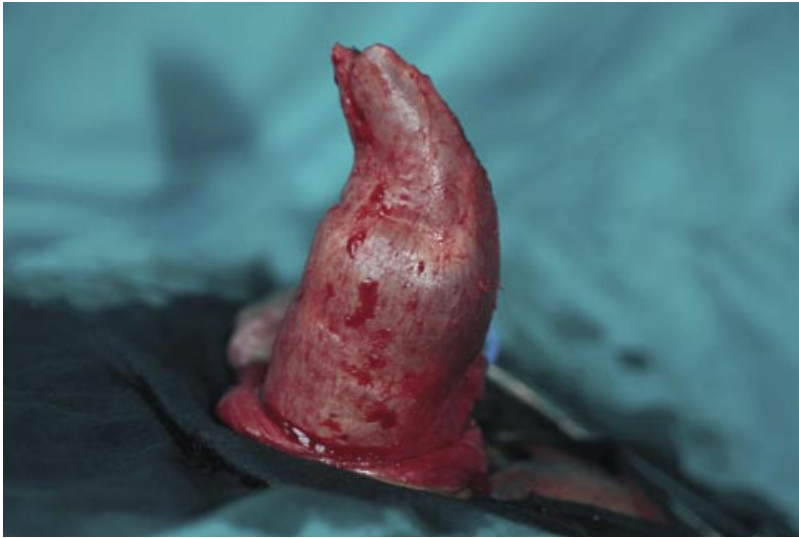

FIGURA 6B). Desmontaje del pene - el levantamiento del glande permite el abordaje de la deformidad apical. Estrechamiento exagerado de las puntas corporales.

Se recomienda a los pacientes la utilización del dispositivo de estiramiento del pene aproximadamente 2-3 semanas después de la operación y el dispositivo de vacío 1-2 semanas más tarde para evitar la retracción del injerto y el consiguiente acortamiento de pene y reproducción de la curvatura.

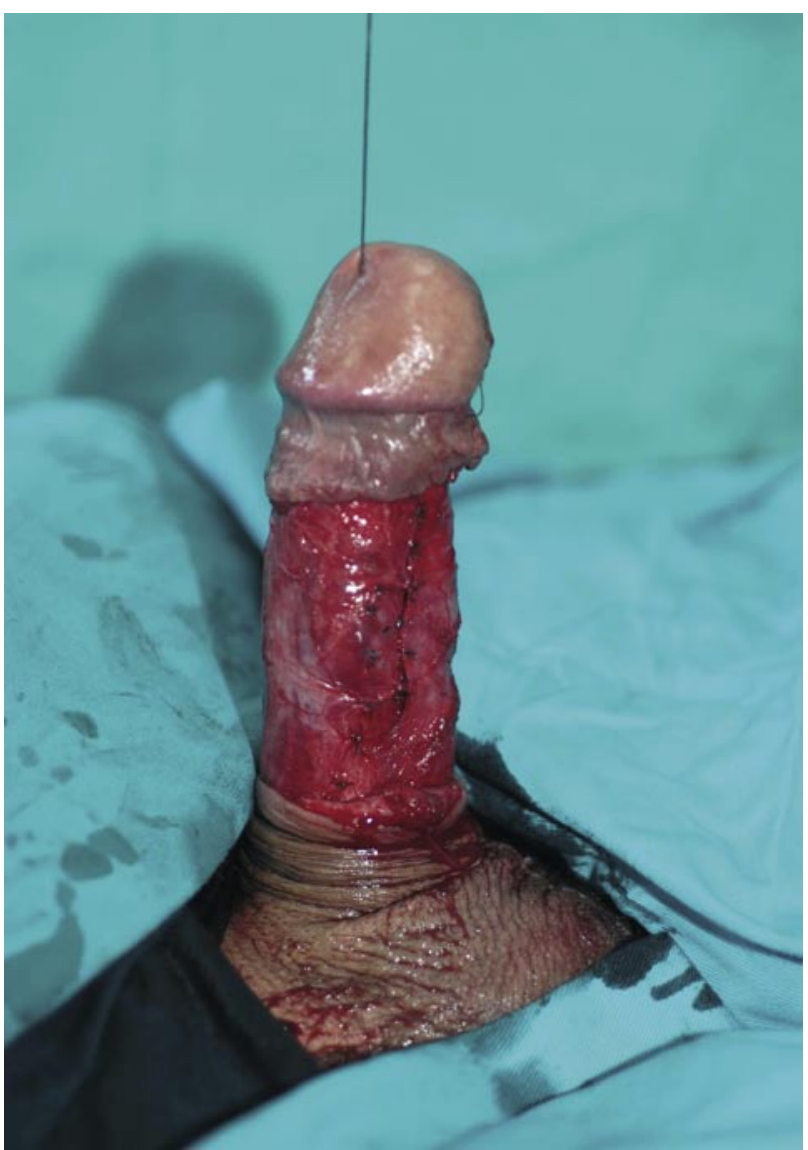

FIGURA 6D). El nuevo montaje del pene proporciona su contorno normal. 


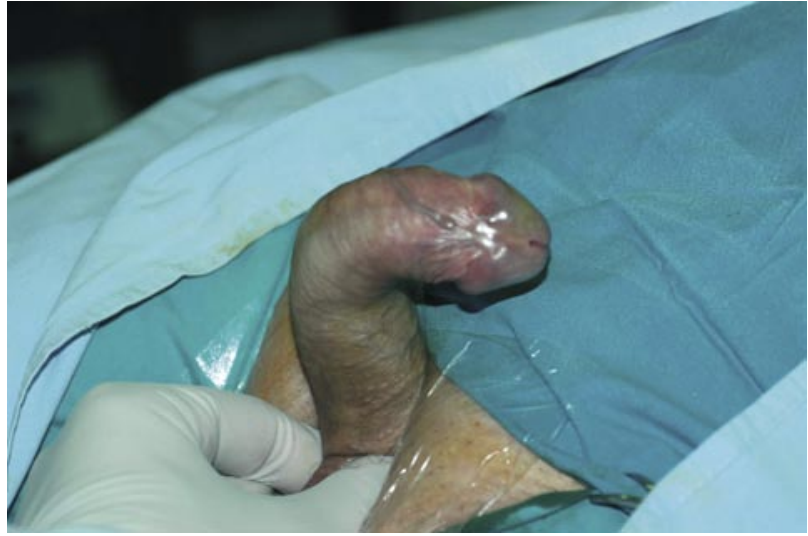

FIGURA 7. Injerto Complejo - Deformidad "Corkscrew":

A). Pene con deformidad "corkscrew".

Se aplicó fisioterapia 4-6 meses después de la cirugía.

También aconsejamos el uso del dispositivo de vacío en pacientes con implante de prótesis de pene.

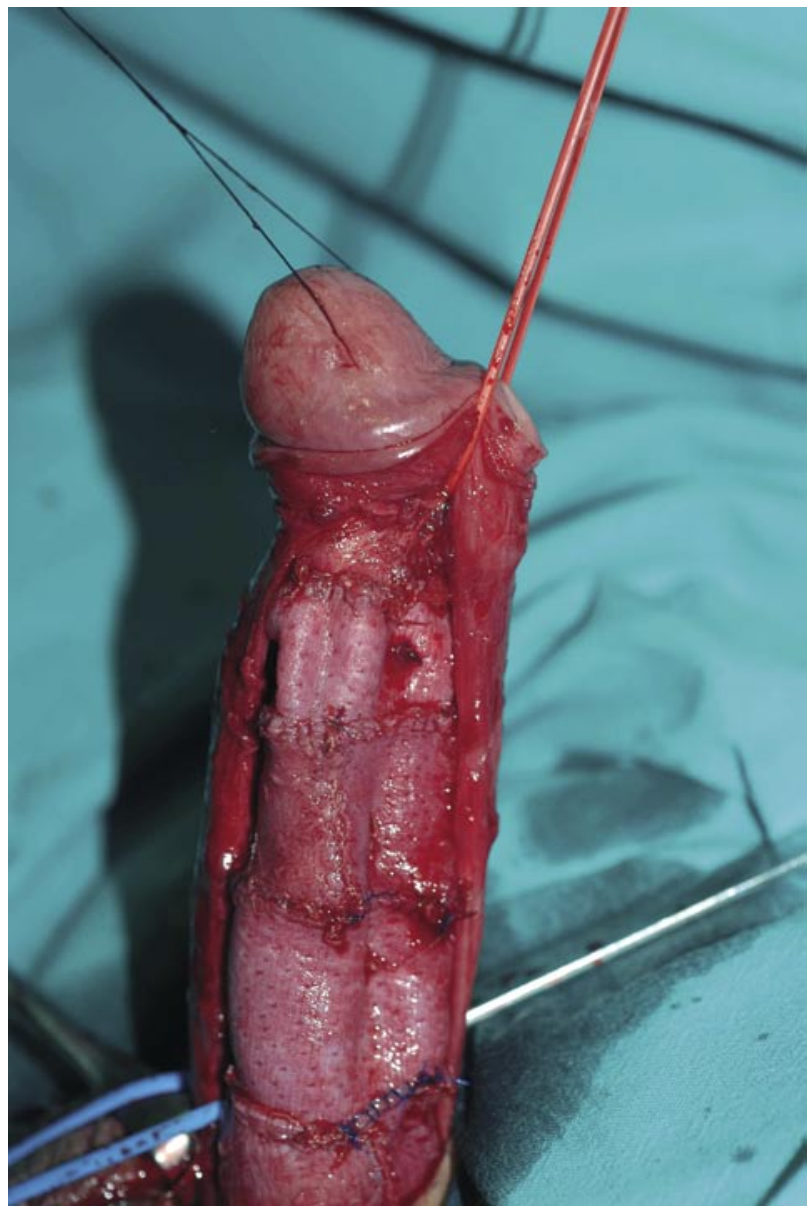

FIGURA 7C). Tallaje completo del pene por medio de varios injertos longitudinales y transversales.

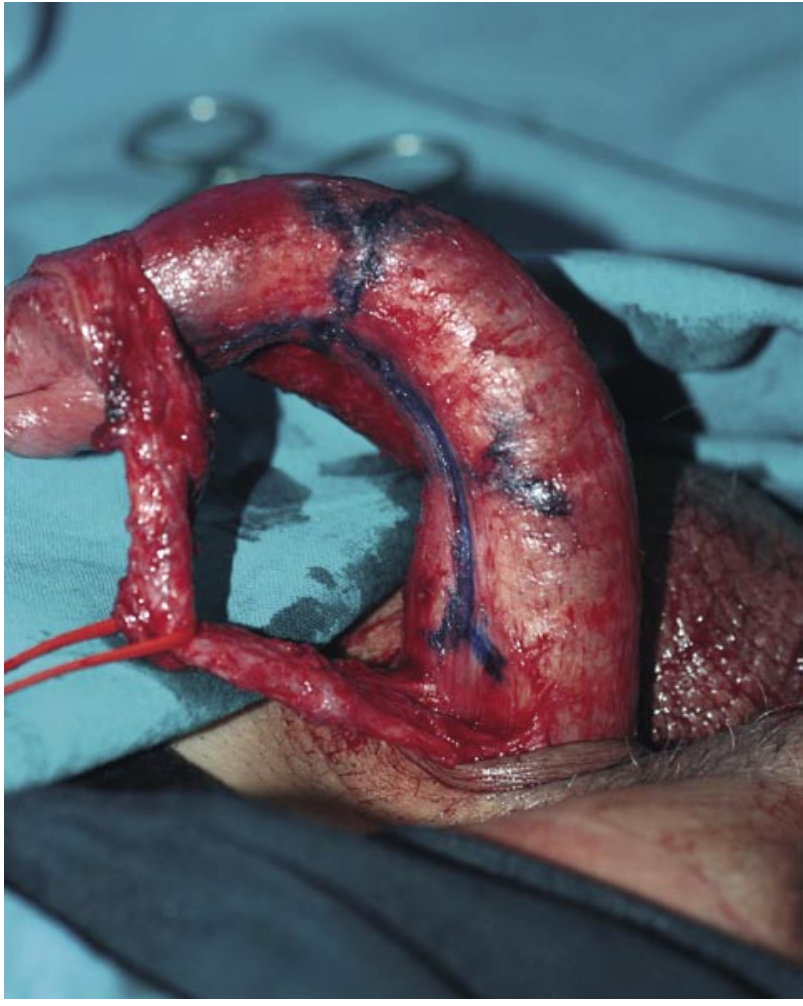

FIGURA 7B). Medida geométrica compleja en erección completa.

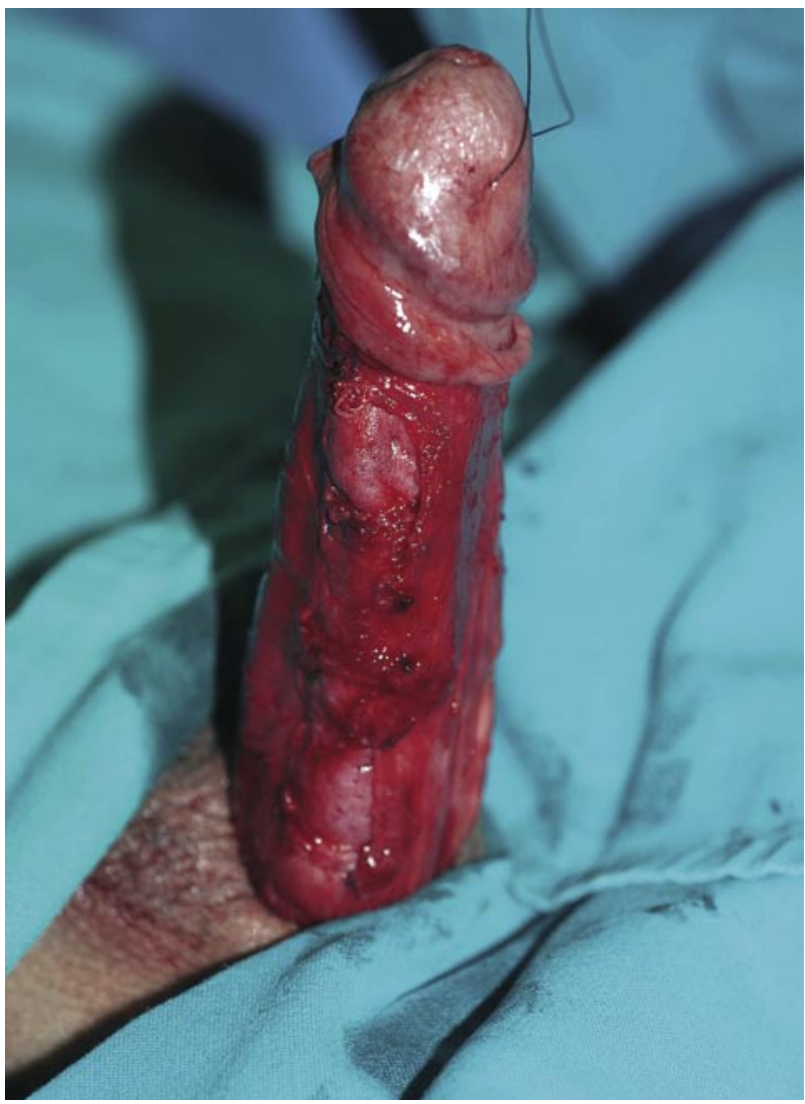

FIGURA 7D|. Implantación de una prótesis maleable. 


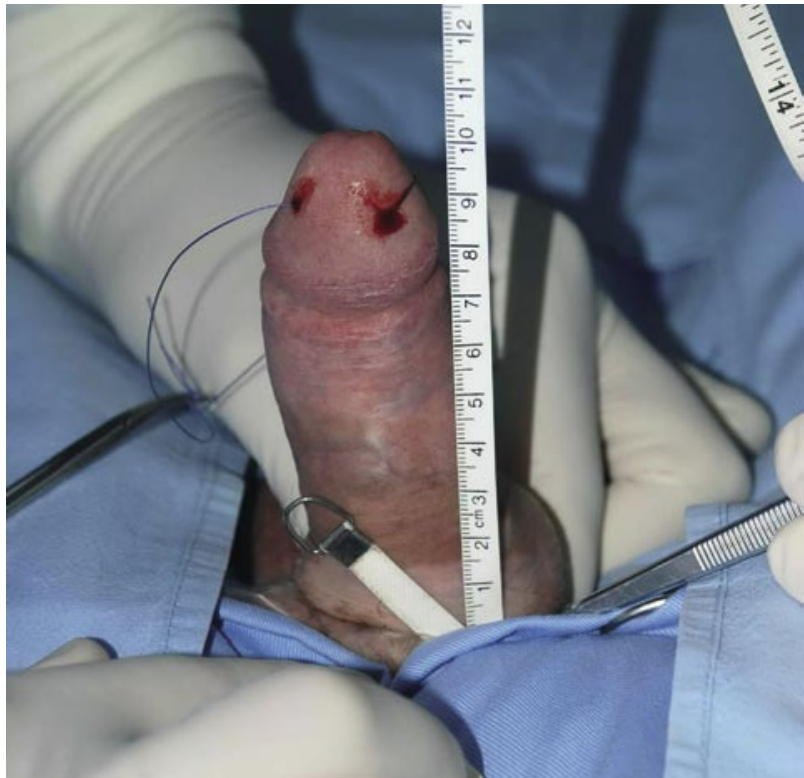

FIGURA 8. Alargamiento cavernoso - dos incisiones circulares, injerto e implante de prótesis maleable: A). Acortamiento del pene sin ninguna deformidad.

\section{RESULTADOS}

El seguimiento fue de 6 a 25 meses (media 15 meses). No hubo ninguna complicación intraoperatoria. Los pacientes fueron re-evaluados 1 semana, $1,3,6$ y 12 meses después de la cirugía. La ganancia media de longitud del pene sin prótesis fue $2,8 \mathrm{~cm}$ $(1,5-4,2)$ y $3,2 \mathrm{~cm}$ con prótesis $(2-4.5 \mathrm{~cm})$. En todos los casos de estrechamiento del cuerpo cavernoso, se restableció el contorno normal del pene antes de la enfermedad. Se reprodujo la curvatura en el $5 \%$ de los pacientes ( 5 pacientes), pero dentro de un rango bajo de curvatura $\left(<15^{\circ}\right)$ y ninguno de ellos necesitó

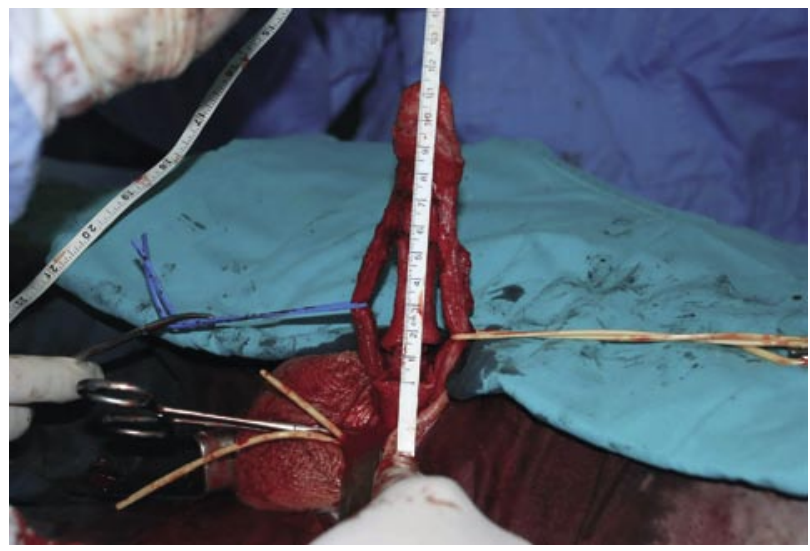

FIGURA 8C). Segunda incisión circular con injerto $(1,5 \mathrm{~cm})$.

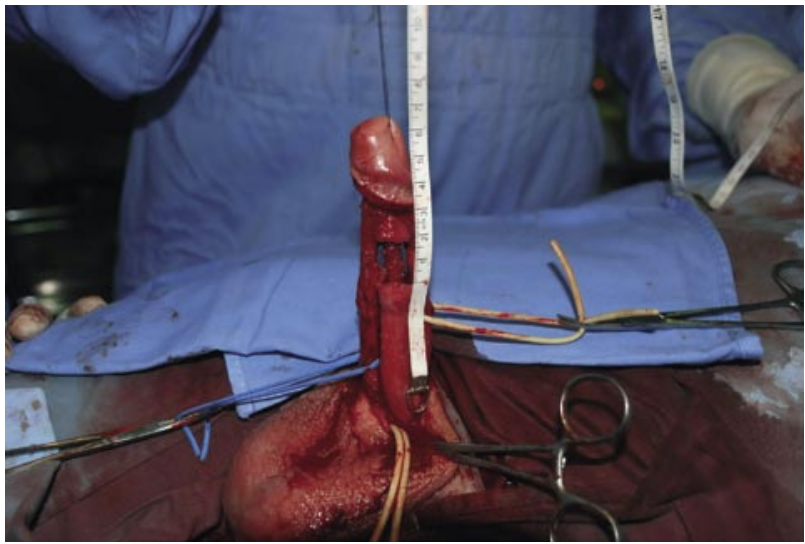

FIGURA 8B). Se movilizan el haz neurovascular y la uretra. Primera incisión circular y creación del defecto tunical $(1,5 \mathrm{~cm})$ con preservación del tejido eréctil e injerto.

reintervención. Veinticuatro pacientes $(24 \%)$ informaron de hipostesia transitoria y sensibilidad orgásmica menor, que se recuperó espontáneamente al cabo de 3-6 meses.

La ecografía Doppler postoperatoria en los pacientes sin implante de pene (61 pacientes, 62\%) mostraron los mismos o mejores valores de velocidad sistólica máxima (VSM) y velocidad diastólica final (VDF) (Tabla II). Se evaluó la actividad sexual del paciente mdiante cuestionario IIEF-5 (4). En los pacientes que se sometieron a cirugía sin implante de prótesis de pene (61 pacientes), la puntuación del IIEF-5 postoperatoria fue 22-24 (sin DE), en 54 pacientes $(89 \%)$, mientras que en 7 pacientes (11\%) fue 19-21 (leve). 54 pacientes tuvieron relación sexual normal 3-6 meses después de la cirugía, y 7 pacientes (1 1\%) con puntuación de $19-21 \mathrm{~m}$ después de la operación

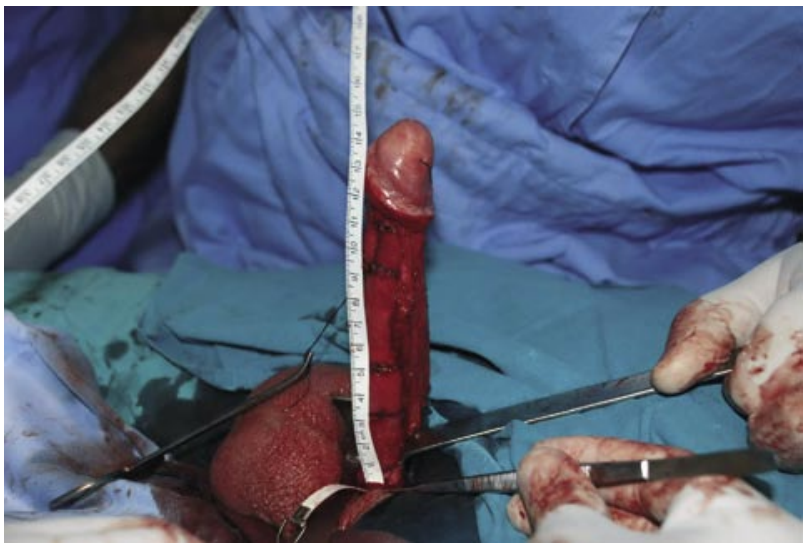

FIGURA 8D). El aumento máximo de la longitud está limitado por la longitud del haz neurovascular. 


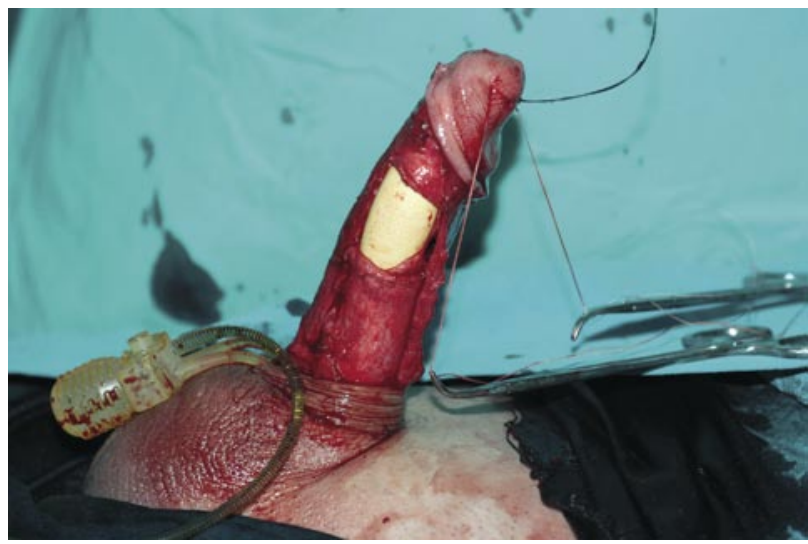

FIGURA 9 Alargamiento cavernoso - una incisión circular, injerto e implante de prótesis inflable:

A). Alargamiento del cuerpo cavernoso con una incisión circular, injerto e implante de prótesis inflable (AMS 700 MS LGX).

utilizaron inhibidores PDE5. La DE de novo ocurrió en 6 pacientes y en 6 pacientes tuvieron progresión de la enfermedad. La satisfacción general de los pacientes con el resultado del procedimiento quirúrgico fue: $5 \%$ no está satisfecho, $31 \%$ satisfecho, $64 \%$ muy satisfecho. En los casos de implante de prótesis de

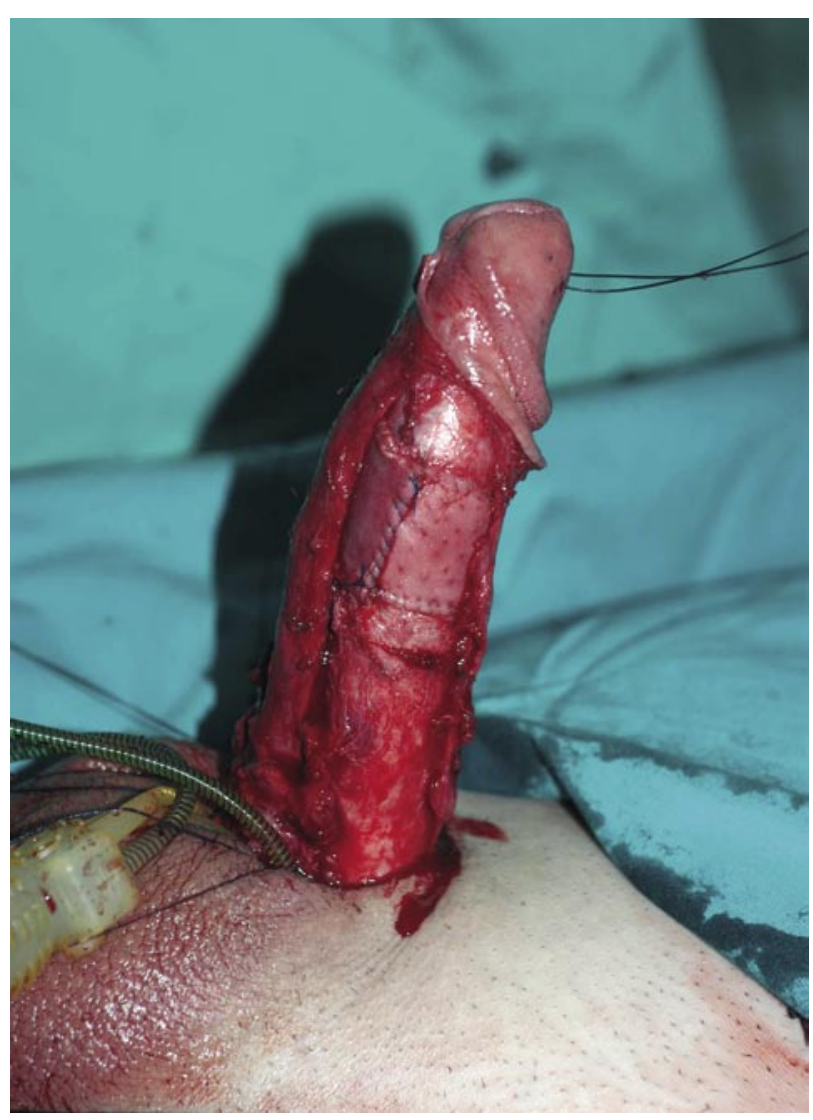

FIGURA 9B). Se observa la unión del injerto circular. pene la satisfacción con la vida sexual de los pacientes $(98 \%)$ y sus parejas $(100 \%)$ fue mayor que $\sin$ prótesis (92\%, 93\%) (Tabla III).

Se restableció la relación sexual en todos los pacientes con implantes de pene, lo que significó mejoría en la satisfacción y la autoestima. Un paciente con diabetes grave, tuvo infección, 10 meses después de la cirugía. Este caso se resolvió con éxito tres meses más tarde, con re-implantación de la prótesis de pene.

\section{DISCUSIÓN}

La búsqueda del tratamiento ideal de la enfermedad de Peyronie grave constituye un gran desafío. El consentimiento informado preoperatorio es fundamental para la preparación de los pacientes con enfermedad de Peyronie para la cirugía. Muchos de estos hombres están deprimidos, tienen una acusada reducción de la autoestima, y muchas veces tienen expectativas inalcanzables con respecto al resultado de la reconstrucción quirúrgica (4). Por lo tanto, se debe iniciar una conversación detallada sobre la gravedad de la curvatura, aceptando el objetivo de conseguir que el paciente sea mantenga la función con el pene recto, que definimos como con una incurvación menor a 15 grados. Otros posible efectos de la cirugía pueden ser la pérdida de longitud del pene, la disminución de la rigidez, sensación sexual y problemas con el orgasmo y la eyaculación. El paciente debe ser informado de que, en algunos casos, la progresión de la enfermedad es inevitable, y no existe ningún parámetro exacto para predecirlo. La progresión suele aparecer en varios meses o en 2 o 3 años.

La variedad de técnicas muestra que no existe consenso con respecto a cualquier procedimiento único que satisfaga las necesidades de la mayoría de los pacientes. El injerto de albugínea revela diferentes riesgos y complicaciones potenciales, incluyendo: $D E$, progresión de la enfermedad, disminución de la sensibilidad orgásmica, acortamiento del pene, incurvación residual o recurrente.

Se han utilizado diferentes injertos en la cirugía de la enfermedad de Peyronie, incluyendo el injerto autólogo (dermis, vena, fascia temporal, túnica vaginal, mucosa bucal) y no autólogo (material procedente de cadáveres humanos preparados, pericardio, dermis, fascia lata, duramadre, e injertos animales transformados - xenoinjertos: pericardio bovino, submucosa del intestino delgado porcino, matriz dérmica acelular porcina). 
Las desventajas de injerto autólogo son: mayor tiempo quirúrgico, aumento de la morbilidad y la cicatriz en la zona donante del injerto, la cantidad de tejido puede ser insuficiente, especialmente en los autoinjertos de venas y crura del pene. En el pasado, la experiencia personal con pericardio de cadáveres humanos, pericardio bovino y submucosa del intestino delgado porcino, mostraron muchas deficiencias. Los resultados postoperatorios no dependen exclusivamente del tipo de injerto utilizado, sino también sobre el tipo de técnica quirúrgica. Sin embargo, no existe injerto ideal, disponible, pero el avance en la calidad de los materiales de injerto es evidente. En los dos últimos años, hemos utilizado únicamente matriz de colágeno dérmico acelular porcina (InteXen LP, AMS).

Este tipo de injerto demostró ser el más adecuado para la sustitución de los grandes defectos tunicales. Está listo para usar, disponible en varios tamaños, tiene buena resistencia a la tracción con el espesor y consistencia similar a la túnica albugínea y proporciona una excelente estabilidad del injerto al cuerpo cavernoso. No produce reacción inflamatoria y es muy resistente a la infección. La contración de este injerto es pequeña si se aplica correctamente fisioterapia postoperatoria. Los agujeros, dónde estaban los folículos pilosos, proporcionan la incorporación rápida de los vasos sanguíneos en el injerto que induce la creación de nuevo tejido fibroso.

La mayoría de los procedimientos que utilizan injertos empíricos, con pequeños injertos ocasionan incurvación residual, a veces incluso acortamiento en lugar de alargamiento de pene (6-8) (Tabla IV). Nuestra técnica se basa en el cálculo geométrico del lugar de la incisión y la forma y tamaño del injerto (9-11). Hemos introducido varias modificaciones y adiciones a esta técnica con el objetivo de lograr la restauración de la forma y el tamaño del pene antes de la enfermedad: injerto transversal de forma hexagonal en lugar de rectangular para evitar protuberancias en los extremos laterales de la sutura de los injertos y mejorar el contorno; injerto longitudinal en todos los casos de corrección del estrechamiento del cuerpo cavernoso, para restablecer el contorno normal; injerto doble en caso de que existan dos puntos de flexión; evitar el abultamiento postoperatorio del injerto suturándolo al septum.

La técnica se basa en incisiones tunicales independientemente de las características y localización de la placa. Puede ser utilizada para corregir todo tipo de deformidades. Se ha estandarizado, en todos los casos, la disección del haz neuro vascular, mediante abordaje lateral. Lo mas importante es la preservación completa del mismo. Su disección pue-

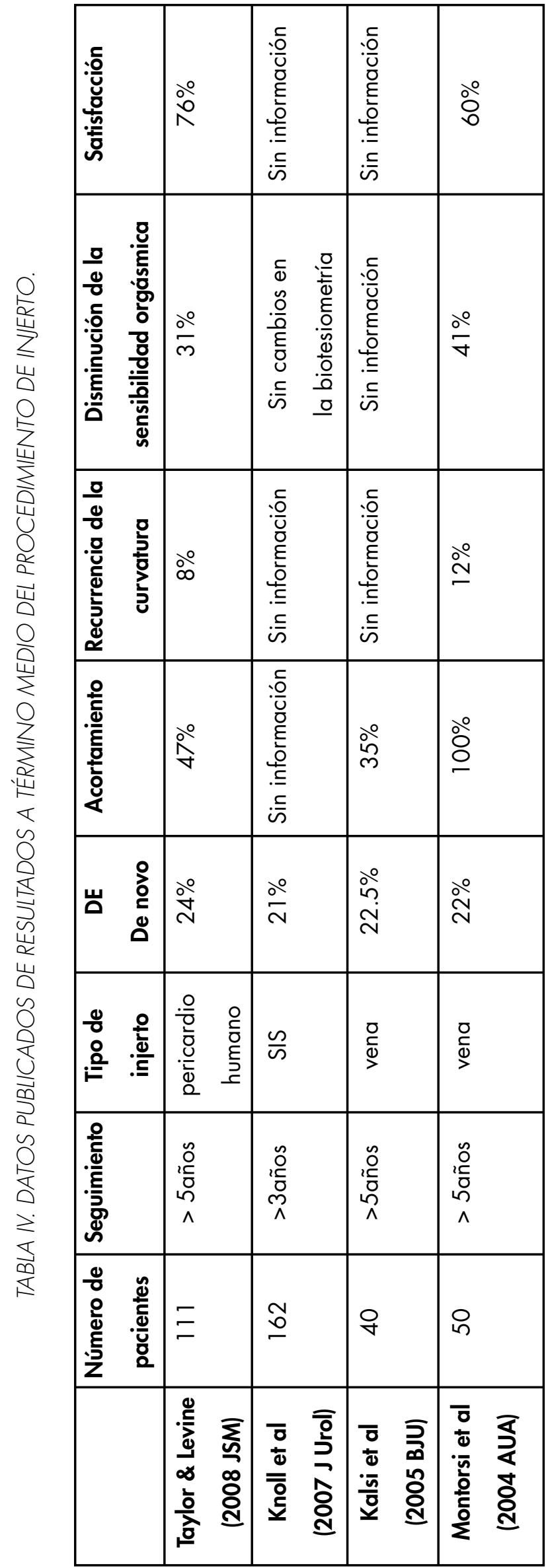


de ser limitada o amplia, lo que permita la elongación máxima, de acuerdo con la gravedad de la enfermedad de Peyronie. El riesgo de lesión de las colaterales de las arterias dorsales se minimiza con menor disección. La ecografía doppler preoperatoria puede definir los lugares de estos colaterales, cuando existan.Para seleccionar el tipo de técnica quirúrgica a utilizar, puede ser muy útil para el cirujano la información sobre la anatomía arterial del pene. Es importante el conocimiento de la existencia de importantes ramas colaterales. Durante la disección del haz neurovascular, se pueden eliminar o preservar esas arterias colaterales si el paciente las tiene en el lugar del punto máximo de curvatura. De lo contrario, si mantenemos estas colaterales, se realizan procedimientos de plicatura con la consecuencia de acortamiento del pene. Schaeffer et al informaron de $44 \%$ de anomalías arteriales, y del $10 \%$ de colaterales distales entre las arterias dorsal y cavernosa (12).

El paciente debe ser informado antes de tomar la decisión acerca de dos diferentes tipos de cirugía. Aconsejamos fervorosamente al paciente aceptar la cirugía de corrección de la curvatura asociada a la implantación simultánea de prótesis cuando el paciente presenta disfunción eréctil.

Kendirci et al, correlacionan la situación vascular con el tipo de deformidad del pene, demostrando una relación entre un tipo de curvatura y la hemodinámica del pene (13). La ecografia dopler color proporciona datos esenciales para la evaluación vascular, insuficiencia arterial y/o disfunción venooclusiva. Los valores de la velocidad sistólica máxima (VSM), mayor de $40 \mathrm{~cm} / \mathrm{sec}$, y el índice de resistencia (IR), superior a 0,9 , se consideran buenos parámetros para el procedimiento de injerto sin implante de prótesis de pene. Los valores de velocidad diastólica final (VDF) menores a $5 \mathrm{~cm} / \mathrm{s}$ indican disfunción veno-oclusiva. Se consideran normales los valores de VDF mayores de $5 \mathrm{~cm} / \mathrm{s}$. Durante o poco después de ecografía, se comparó, de forma objetiva, la rigidez del pene con la rigidez auto-informada. Esto permite una evaluación más objetiva de la rigidez. Si la rigidez es menor con la prueba de inyección intracavernosa (IIC), se presionan ambas cruras del pene sobre el púbis óseo para obtener la máxima rigidez con el fin de evaluar la deformidad real del pene, mientras que por otro lado para evaluar la rigidez axial se oprime el glande imitando un intento de penetración.

La disección de la línea media del haz neurovascular, que algunos autores utilizan, no es adecuada para la corrección de los casos más graves (14). La creación de una erección completa es de gran importancia para la aplicación correcta de los prin- cipios geométricos, y la determinación de los puntos apropiados para la incisión de la túnica albugínea. El principio básico para el alargamiento del cuerpo cavernoso es igualar el lado más corto con el lado más largo. La diferencia entre los dos lados define el ancho del defecto. El enderezamiento completo del pene se verifica por tracción del pene después de las incisiones finales en la túnica en el septo, y la disección de la albugínea del tejido eréctil de los cuerpos cavernosos. Su estructura es similar a la uretra esponjosa, y aunque es muy elástica, se moviliza también a fin de enderezar el pene y prevenir el abultamiento del injerto. Si el haz neurovascular es un factor limitante para enderezar el pene, su disección puede ser ampliada. La uretra no es factor limitante para el alargamiento debido a su elasticidad. Sólo se utilizó una incisión e injerto cuando el pene estaba curvado en un punto.

Si hay dos puntos de flexión, son necesarias dos incisiones y dos injertos para enderezar el pene. Debe evitarse, siempre que sea posible la plicatura complementaria, porque pone en peligro las ventajas del principio geométrico en la restauración completa de enderezamiento, alargamiento y ensanchamiento del pene. En todos los casos en los que esté indicado el implante de prótesis de pene, se realizará en primer lugar la restauración de la forma del pene mediante injerto. En casos, con acortamiento aislado del pene, la incisión circular completa y el injerto proporcionan alargamiento corporal real. La técnica presentada es efectiva para la corrección de todos los tipos de deformidades en el pene, independientemente de las características de la placa.

El dolor postoperatorio de pene es una queja común y corresponde a la recuperación del nerviosa sensorial después del estiramiento quirúrgico de los haces neurovasculares. El repos y los analgésicos resultan ser un buen tratamiento, ya que en la mayoría de los casos, se debe a un fenómeno temporal.

La fisioterapia postoperatoria se inicia el segundo o tercer día postoperatorio con inhibidores PDE5 (durante 3-4 semanas) y continua con estiramientos (2-3 semanas después de la operación) y uso del dispositivo de vacío (1-2 semanas después) para evitar la retracción del injerto y permitir la curación en ambos estados flácido y erecto 15). Deben utilizarse durante todo el período de cicatrización entre $4-6$ meses $(16,17)$.

\section{CONCLUSIONES}

El tratamiento quirúrgico de la enfermedad de Peyronie grave basado en el principio geométrico 
es eficaz para la reconstrucción extensa del cuerpo cavernoso del pene, para corregir el acortamiento y estrechamiento grave del pene, dando como resultado una ganancia en la longitud máxima del pene y la restauración del contorno, independientemente de las características de la placa. La forma del pene y el restablecimiento del tamaño son muy importantes para la autoestima y la satisfacción global del paciente.

\section{BIBLIOGRAFÍA y LECTURAS RECOMENDADAS ( ${ }^{*}$ lectura de interés $y^{* *}$ lectura fundamental)}

1. Rochelle JC, Levine LA. Survey of primary care physicians and urologist regarding Peyronies disease. J Urol 2005; 173 (Supl). 254 (Abstract 941)

*2. Christopher JS, Chelsea M, Ridwan S. Peyronies disease: the epidemiology, aetiology and clinical evaluation of deformity. BJU Int2005; 95:72932.

*3. Usta MF, Bivalacqua TJ, Tokatili Z et al. Stratification of penile vascular pathologies in patients with Peyronies disease and in man with erectile dysfunction according to age: a comparative study. J Urol 2004;172:259-62.

4. Nelson CJ, Diblasio C, Kendirci M, Hellstrom W, Ghuring P, Mulhall JP. The Chronology of Depresion and Distres in Men with Peyronie's Desease. J Sex Med, 2008;5:1985-90.

*5. Rosen RC, Cappelleri JC, Smith MD, Lipsky J, Peña BM. Development and evaluation of an abridged, 5-item version of the International Index of Erectile Function (IIEF-5) as a diagnostic tool for erectile dysfunction. Int J Impot Res. 1999;11(6):319-26.

6. Kalsi J, Minhas S, et al. BJU Int 2005;95:102933.
*7. Montorsi F, Salonia. Annual Meeting of the American Urological Association. 2004.

8. Taylor FL, and Levine LA. J Sex Med 2008;5:22212228.

**9. Egydio PH, Lucon AM, Arap S. Treatment of Peyronie's disease by incomplete circumferential incision of the tunica albuginea and plaque with bovine pericardium graft. Urology. 2002 Apr;59(4):570-4.

*10. Egydio PH. Surgical treatment of Peyronie's disease: choosing the best approach to improve patient satisfaction. Asian J Androl. 2008 Jan;10(1):15866.

**11. Egydio PH, Lucon AM, Arap S. A single relaxing incision to correct different types of penile curvature: surgical technique based on geometrical principles. BJU Int. 2004 Nov;94(7):1147-57.

*12. Schaeffer EM, Jarow JP Jr, Vrablic J, Jarow JP. Duplex ultrasonography detects clinically significant anomalies of penile arterial vasculature affectingsurgical approach to penile straightening. Urology 2006;67:166-9.

*13. Kendirci M, Nowfar S, Gur S, Jabren GW, Sikka SC, Hellstrom WJ. The relationship between the type of penile abnormality and penile vascular status in patients with Peyronie's disease. Urology 2005; 174:632-5.

14. Hellstrom W. Medical management of Peyronie's disease. J Androl 2009 Jul-Aug;30(4):397-405.

15. Levine LA, Newell M, Tailor FL. Penile traction therapy for treatment of Peyronie's disease: A single center pilot study. J Sex Med 2008;5:146873.

**16. Moncada-Iribarren I, Jara J, Martinez-Salamanca JI, Cabello R, Hernandez C. Managing penile shortening after Peyronie's disease surgery. J Urol 2007;177(4supl):252.

17. Tornehl CK, Carson CC. Peyronie's disease surgery: which technique for which patient. Contemporary Urology 2004;vol 16: no6. 